\title{
Variational approach to the dilute Bose gas
}

\author{
M. Bijlsma and H. T. C. Stoof \\ University of Utrecht, Institute for Theoretical Physics, Princetonplein 5, P.O. Box 80.006, 3508 TA Utrecht, The Netherlands
}

(Received 16 April 1996)

\begin{abstract}
We study the weakly interacting Bose gas in both two and three dimensions using a variational approach. In particular we construct the thermodynamic potential of the gas to within ladder approximation and find by minimization an accurate mean-field description of the dilute Bose gas. Using spin-polarized atomic hydrogen as a specific example, we obtain an improved description of the Bose-Einstein condensed phase in three dimensions, and a signature of a phase transition in two dimensions. The latter cannot be found by a straightforward application of perturbation theory around the ideal Bose gas. [S1050-2947(97)03801-8]
\end{abstract}

PACS number(s): 03.75.Fi, 64.10.+h, 67.65.+z, 67.40.-w

\section{INTRODUCTION}

The theoretical study of a three-dimensional dilute Bose gas in the regime where quantum degeneracy effects play a role has a long history [1], and was first important for a microscopic understanding of the $\lambda$ transition in liquid ${ }^{4} \mathrm{He}$, although quantitative predictions for this strongly interacting system cannot be obtained by these means. The latter is, however, not true for magnetically trapped quantum gases [2], which explains why the phenomenon of Bose-Einstein condensation, accompanied by the occurrence of superfluidity, is still an active field of research today. Moreover, it turns out that there is no completely satisfying theory for these systems $[3,4]$, since existing treatments, which are based on a Bogoliubov kind of approach, fail, for instance, to show the correct order of the phase transition and yield a first-order transition to the condensed phase $[5,6]$ instead of the second-order transition expected from the theory of critical phenomena. In this paper we will develop a variational approach and show, for example, that a small but essential change in the Bogoliubov theory can resolve this issue. This is one of the strong points of the variational method.

The two-dimensional dilute Bose gas has become subject of study only more recently when it was realized that this system is in the same universality class as the $X Y$ model [7] and should therefore exhibit a Kosterlitz-Thouless phase transition [8]. Physically this implies that there is no macroscopic occupation of one single quantum state, but there is nevertheless a transition to a superfluid state through the mechanism of the binding of vortices with opposite vorticity. This mechanism causes the superfluid density $n_{s}$ to show a universal discontinuity such that $n_{s} \Lambda^{2}=4$ at the critical temperature [9], where $\Lambda=\left(2 \pi \hbar^{2} / m k_{B} T\right)^{1 / 2}$ is the thermal de Broglie wavelength and $m$ is the mass of the particles in the system. The study of the two-dimensional Bose gas starting from a microscopic Hamiltonian was initiated by Popov [10]. In his approach, which corresponds to a one-loop calculation and is based on the introduction of a so-called quasicondensate, the superfluid density exhibits no jump, but instead goes to zero in a continuous fashion. In addition, it has been shown $[11,12]$ that the range of validity of Popov's approach is by no means attainable in the experimental setups envisaged at present. Therefore, we have recently extended this work using the ladder or $T$-matrix approximation which should be sufficiently accurate at low densities since it includes all two-body processes [12]. In this manner we correctly found a jump in the superfluid density, but unfortunately, due to infrared divergences, that the magnitude of the quasicondensate also had to be adjusted such that the jump attained the universal value predicted by Nelson and Kosterlitz using renormalization-group methods [9]. This latter aspect of our work shows that the ladder approximation breaks down close to the critical temperature, and needs improvement in the critical region. This problem will be addressed in a future publication. In this paper we will also apply our variational approach developed for the three-dimensional case to the two-dimensional Bose gas, because in this way we can in principle also resolve the infrared divergences, making it an interesting approach for studying the twodimensional system.

The classical experimental realization of a dilute Bose gas in which one can try to observe either Bose-Einstein condensation or the Kosterlitz-Thouless transition is spin-polarized atomic hydrogen. Although there is no fundamental reason for Bose-Einstein condensation not to be attainable in this system, the actual achievement of the critical condition is an extremely difficult task due to the inherent instability of the atomic hydrogen gas against spin-flip processes, which lead to particle loss and in general also cause heating of the gas sample. The two-dimensional configuration of course also suffers from these drawbacks, but the critical conditions are more favorable than for the three-dimensional case and, as of the mid 1980s, several experimental groups have therefore been pursuing the observation of the Kosterlitz-Thouless transition [13-16]. In these experiments one uses liquid ${ }^{4} \mathrm{He}$ films to confine the hydrogen atoms in one spatial direction, hence realizing an effectively two-dimensional Bose gas.

Other experimental realizations of a dilute Bose gas in which one can observe quantum degeneracy effects are lasercooled alkali-metal atoms and excitons. Since evaporative cooling was recently shown to work for magnetically trapped alkali-metal atoms like $\mathrm{Rb}$ and $\mathrm{Na}[17,18]$, advances toward the observation of Bose-Einstein condensation were particularly fast, and recently actual observations of a Bose-Einstein condensate in ultracold atomic ${ }^{87} \mathrm{Rb},{ }^{7} \mathrm{Li}$, and ${ }^{23} \mathrm{Na}$ gases have been reported [19-21]. Recent developments in molecular-beam-epitaxy technologies have also led to op- 
portunities to reach the critical conditions for the KosterlitzThouless transition in a two-dimensional (dumbbell) exciton gas confined by a double quantum-well structure [22]. The three-dimensional exciton gas in, e.g., pure $\mathrm{Cu}_{2} \mathrm{O}$ crystals has a somewhat longer history [23]. It has even been claimed that the condensation transition was already observed in such a system [24]. However, mainly due to the lack of a convincing interpretation of the obtained experimental data, there is no decisive proof of this fact as yet.

As mentioned above, here we will first study the dilute Bose gas in general by making use of a variational approach, and then take spin-polarized atomic hydrogen as a specific example. A variational calculation is a conceptually simple tool $[25,26]$ that has been applied to such various subjects as, for instance, the investigation of the triviality of $\lambda \phi^{4}$ field theories in different spacetime dimensions [27], investigation of the phase diagram of the $O(N)$ vector model [28], and construction of the ground state of the Sine-Gordon model [29].

So the idea of a variational calculation is not new, but it has, to the best of our knowledge, never been applied to the dilute Bose gas. Therefore, the outcome of such a calculation is interesting in its own right. Moreover, it turns out that it gives new insight into a number of problems present in the conventional treatments of the dilute Bose gas, and into the way to resolve them. This will be explained in more detail below.

The basic idea is to make a Gaussian ansatz for the ground-state wave functional and its excitations, which amounts to making an ansatz for the dispersion of the quasiparticles in the system, and then to calculate the approximate thermodynamic potential $\Omega=\Omega_{\mathrm{tr}}+\left\langle\mathcal{H}-\mathcal{H}_{\mathrm{tr}}\right\rangle_{\mathrm{tr}}$. Here $\mathcal{H}_{\text {tr }}$ is a trial Hamiltonian that has trial wave functionals as eigenstates, $\Omega_{\mathrm{tr}}=-k_{B} T \ln \left(\operatorname{Tr}\left[e^{-\beta\left(\mathcal{H}_{\mathrm{tr}}-\mu \mathcal{N}\right)}\right]\right)$ and \langle\rangle$_{\mathrm{tr}}$ is the corresponding grand-canonical thermal average. One subsequently minimizes this thermodynamic potential with respect to the variational parameters that are in the Gaussian wave functionals, and with respect to the expectation value of the field of interest.

In the case of the dilute Bose gas, the results obtained in this manner have to be used in the equation of state, which makes, for example, a determination of the critical temperature possible. This procedure is a mean-field approach in the sense that no fluctuations in the expectation value of the field are taken into account. However, it is nonperturbative, and clearly goes beyond the one-loop level. The main advantage of using a variational approach in two dimensions is that the notorious infrared divergences are automatically circumvented because the dispersion relation of the particles will in general display a gap. This gap is analogous to the (arbitrary) infrared cutoff $k_{0}$ that Popov introduced to define the quasicondensate $n_{0}\left(k_{0}\right)$. Note, however, that in the variational procedure minimization of the thermodynamic potential will fix the magnitude of the gap. Hence there will be no adjustable parameter left in the calculation, as was the case in our previous treatment of the two-dimensional dilute Bose gas, where we had to use the universal jump relation to determine the infrared cutoff $k_{0}$ [12]. This convenient feature strongly motivated us also to study the two-dimensional system within the framework of a variational calculation.
Our Gaussian ansatz will also introduce a condensate in two dimensions. Of course, the existence of a true condensate in two dimensions is principally forbidden by the Mermin-Wagner-Hohenberg theorem [30]. However, using trial wave functionals with a broken symmetry in a variational approach can still be useful, because even if the form of the trial wave functionals deviates considerably from the exact wave functionals describing the phase below the critical temperature, the estimate of the total energy of the system can be extremely good.

The organization of this paper is as follows. In Sec. II we derive the desired expression for the thermodynamic potential $\Omega$, and we show in particular that minimization with respect to the variational parameters automatically introduces the many-body $T$ matrix into the problem. However, it turns out that the variational principle applied to the microscopic Hamiltonian does not yield a completely consistent picture. This problem is subsequently resolved by applying the variational principle to an appropriate effective Hamiltonian. After this improvement we are ready to apply the theory to atomic hydrogen. The results are presented in Sec. III. In Sec. III A we consider the three-dimensional system, and Sec. III B is devoted to the two-dimensional system. Our approach indicates the onset of a new phase in the latter case which cannot be found by an application of perturbation theory around the ideal Bose gas. However, we are not able to describe the properties of this phase by our variational method, but argue that it corresponds to the superfluid bound-vortex state. In Sec. IV we draw some conclusions from our work.

\section{THERMODYNAMIC POTENTIAL}

We study the dilute Bose gas using the functional integral formulation of the grand-canonical partition function. Starting with $Z_{\mathrm{gr}}=\operatorname{Tr}\left[e^{-\beta(\mathcal{H}-\mu \mathcal{N})}\right]$, where $\mathcal{H}$ is the microscopic Hamiltonian of the gas that in the language of second quantization can be expressed in the creation and annihilation operators $\psi^{\dagger}(\mathbf{x})$ and $\psi(\mathbf{x})$, we can write

$$
Z_{\mathrm{gr}} \equiv e^{-\beta \Omega}=\int d\left[\psi^{*}\right] d[\psi] \exp \left\{-\frac{1}{\hbar} S\left[\psi^{*}, \psi\right]\right\},
$$

after using the closure relation of the eigenstates of the annihilation operator at each imaginary time $\tau$ [31]. The functional integral is over $c$-number fields $\psi^{*}(\mathbf{x}, \tau)$ and $\psi(\mathbf{x}, \tau)$, periodic in imaginary time over $\hbar \beta=\hbar / k_{B} T$. The Euclidian action for the dilute Bose gas is given by

$$
\begin{aligned}
S\left[\psi^{*}, \psi\right]= & \int_{0}^{\hbar \beta} d \tau\left(\int d \mathbf{x} \psi^{*}(\mathbf{x}, \tau)\right. \\
& \times\left[\hbar \frac{\partial}{\partial \tau}-\frac{\hbar^{2} \nabla^{2}}{2 m}-\mu\right] \psi(\mathbf{x}, \tau) \\
& +\frac{1}{2} \int d \mathbf{x} \int d \mathbf{x}^{\prime} \psi^{*}(\mathbf{x}, \tau) \psi^{*}\left(\mathbf{x}^{\prime}, \tau\right) \\
& \left.\times V\left(\mathbf{x}-\mathbf{x}^{\prime}\right) \psi\left(\mathbf{x}^{\prime}, \tau\right) \psi(\mathbf{x}, \tau)\right),
\end{aligned}
$$


with $\mu$ the chemical potential and $V\left(\mathbf{x}-\mathbf{x}^{\prime}\right)$ the effectively repulsive interaction potential, meaning that the corresponding scattering length $a$ is positive.

Expanding the fields in Fourier modes through

$$
\psi(\mathbf{x}, \tau)=\frac{1}{(\hbar \beta V)^{1 / 2}} \sum_{\mathbf{k}, n} a_{\mathbf{k}, n} e^{i\left(\mathbf{k} \cdot \mathbf{x}-\omega_{n} \tau\right)}
$$

and the complex conjugate expression for $\psi^{*}(\mathbf{x}, \tau)$, we can write the action in momentum space as

$$
\begin{aligned}
S\left[a^{*}, a\right]= & \sum_{\mathbf{k}, n}\left(-i \hbar \omega_{n}+\epsilon_{\mathbf{k}}-\mu\right) a_{\mathbf{k}, n}^{*} a_{\mathbf{k}, n} \\
& +\frac{1}{2} \frac{1}{\hbar \beta V} \sum_{\substack{\mathbf{k}, \mathbf{k}^{\prime}, \mathbf{q} \\
n, n^{\prime}, m}} V_{\mathbf{q}} a_{\mathbf{k}+\mathbf{q}, n+m}^{*} a_{\mathbf{k}^{\prime}-\mathbf{q}, n^{\prime}-m}^{*} a_{\mathbf{k}^{\prime}, n^{\prime}} a_{\mathbf{k}, n} .
\end{aligned}
$$

In this equation $\epsilon_{\mathbf{k}}=\hbar^{2} \mathbf{k}^{2} / 2 m$, and $V_{\mathbf{q}}=\int d \mathbf{x} V(\mathbf{x}) e^{-i \mathbf{q} \cdot \mathbf{x}}$ is the Fourier transform of the interaction potential. Since we will be interested in the temperature regime where the spatial extension $\Lambda$ of the hydrogen atoms is much larger than the range of the interaction potential $(a / \Lambda \ll 1)$, there is essentially only $s$-wave scattering taking place in the gas, and we can neglect the momentum dependence of the interaction potential. Therefore we set $V_{\mathbf{q}}=V_{\mathbf{0}}$. The momenta are restricted to either two or three dimensions, and $V$ is the area or volume of the corresponding system. The bosonic Matsubara frequencies $\omega_{n}=2 \pi n / \hbar \beta$ reflect the periodicity of the fields in (imaginary) time.

We now have the basic ingredients, and can start the procedure of variationally calculating the thermodynamic potential $\Omega$. Breaking the symmetry by introducing $\psi(\mathbf{x}, \tau)$ $=\sqrt{n_{0}}+\psi^{\prime}(\mathbf{x}, \tau)$ and expanding the resulting expression, the action up to quadratic order in first instance becomes

$$
\begin{aligned}
S^{(2)}\left[a, a^{*}\right]= & -\hbar \beta \mu n_{\mathbf{0}} V+\frac{1}{2} \hbar \beta n_{\mathbf{0}}^{2} V_{\mathbf{0}} V \\
& +\sum_{\mathbf{k}, n}{ }^{\prime}\left(-i \hbar \omega_{n}+\epsilon_{\mathbf{k}}-\mu+2 n_{\mathbf{0}} V_{\mathbf{0}}\right) a_{\mathbf{k}, n}^{*} a_{\mathbf{k}, n} \\
& +\frac{1}{2} n_{\mathbf{0}} V_{\mathbf{0}} \sum_{\mathbf{k}, n}{ }^{\prime}\left(a_{\mathbf{k}, n}^{*} a_{-\mathbf{k},-n}^{*}+a_{\mathbf{k}, n} a_{-\mathbf{k},-n}\right),
\end{aligned}
$$

where the prime denotes that $\mathbf{k}=\mathbf{0}$ is excluded from the sum. From this we see that we have nontrivial so-called normal and anomalous self-energies, and that in the lowest-order approximation they are, respectively, $\hbar \Sigma_{11}\left(\mathbf{k}, \omega_{n}\right)=2 n_{0} V_{\mathbf{0}}$ and $\hbar \Sigma_{12}\left(\mathbf{k}, \omega_{n}\right)=n_{\mathbf{0}} V_{\mathbf{0}}$. This gives a strong indication of the form of the variational wavefunctional that we have to take to describe the dilute Bose gas correctly, in principal at any temperature. As the choice of Gaussian wave functionals is formally identical to an ansatz for the quadratic trial Hamiltonian $\mathcal{H}_{\text {tr }}$ which they are supposed to diagonalize, we now write the full action as

$$
\begin{aligned}
& S\left[a, a^{*}\right]=-\hbar \beta \mu n_{\mathbf{0}} V+\frac{1}{2} \hbar \beta n_{\mathbf{0}}^{2} V_{\mathbf{0}} V+\sum_{\mathbf{k}, n}{ }^{\prime}\left(-i \hbar \omega_{n}+\epsilon_{\mathbf{k}}-\mu+\hbar \Sigma_{11}\left(\mathbf{k}, \omega_{n}\right)\right) a_{\mathbf{k}, n}^{*} a_{\mathbf{k}, n} \\
& +\frac{1}{2} \hbar \Sigma_{12}\left(\mathbf{k}, \omega_{n}\right) \sum_{\mathbf{k}, n}^{\prime}\left(a_{\mathbf{k}, n}^{*} a_{-\mathbf{k},-n}^{*}+a_{\mathbf{k}, n} a_{-\mathbf{k},-n}\right)+\left(2 n_{\mathbf{0}} V_{\mathbf{0}}-\hbar \Sigma_{11}\left(\mathbf{k}, \omega_{n}\right)\right) \sum_{\mathbf{k}, n}{ }^{\prime} a_{\mathbf{k}, n}^{*} a_{\mathbf{k}, n} \\
& +\frac{1}{2}\left(n_{\mathbf{0}} V_{\mathbf{0}}-\hbar \Sigma_{12}\left(\mathbf{k}, \omega_{n}\right)\right) \sum_{\mathbf{k}, n}{ }^{\prime}\left(a_{\mathbf{k}, n}^{*} a_{-\mathbf{k},-n}^{*}+a_{\mathbf{k}, n} a_{-\mathbf{k},-n}\right)+\left(\frac{n_{\mathbf{0}}}{\hbar \beta V}\right)^{1 / 2} \sum_{\substack{\mathbf{k}^{\prime}, \mathbf{q} \\
n^{\prime}, m}}{ }^{\prime} V_{\mathbf{0}} a_{\mathbf{q}, m}^{*} a_{\mathbf{k}^{\prime}-\mathbf{q}, n^{\prime}-m^{\prime}}^{*} a_{\mathbf{k}^{\prime}, n^{\prime}} \\
& +\left(\frac{n_{\mathbf{0}}}{\hbar \beta V}\right)^{1 / 2} \sum_{\substack{\mathbf{k}, \mathbf{q} \\
n, m}}^{\prime} V_{\mathbf{0}} a_{\mathbf{k}+\mathbf{q}, n+m}^{*} a_{\mathbf{q}, m} a_{\mathbf{k}, n}+\frac{1}{2} \frac{1}{\hbar \beta V} \sum_{\substack{\mathbf{k}, \mathbf{k}^{\prime}, \mathbf{q} \\
n, n^{\prime}, m}}^{\prime} V_{\mathbf{0}} a_{\mathbf{k}+\mathbf{q}, n+m}^{*} a_{\mathbf{k}^{\prime}-\mathbf{q}, n^{\prime}-m}^{*} a_{\mathbf{k}^{\prime}, n^{\prime}} a_{\mathbf{k}, n},
\end{aligned}
$$

where we introduced nontrivial normal and anomalous selfenergies in the first two quadratic terms on the right-hand side of Eq. (6) by simply adding and subtracting

$$
\begin{aligned}
& \sum_{\mathbf{k}, n}{ }^{\prime}\left(\hbar \Sigma_{11}\left(\mathbf{k}, \omega_{n}\right) a_{\mathbf{k}, n}^{*} a_{\mathbf{k}, n}+\frac{1}{2} \hbar \Sigma_{12}\left(\mathbf{k}, \omega_{n}\right)\right. \\
& \left.\quad \times\left(a_{\mathbf{k}, n}^{*} a_{-\mathbf{k},-n}^{*}+a_{\mathbf{k}, n} a_{-\mathbf{k},-n}\right)\right)
\end{aligned}
$$

The quantities $\hbar \Sigma_{11}\left(\mathbf{k}, \omega_{n}\right)$ and $\hbar \Sigma_{12}\left(\mathbf{k}, \omega_{n}\right)$ are now considered as the variational parameters of the trial wave functionals, and are determined from a minimization of the thermo- dynamic potential. As indicated by their arguments, the selfenergies are in principle functions of momentum and frequency. However, since we are dealing with a dilute gas in the limit where only $s$-wave scattering is of importance, we will neglect these dependencies and henceforth omit the arguments. This is a usual step taken in treating the weakly interacting Bose gas. Note, furthermore, that we assume the self-energies to be real, hence $\hbar \Sigma_{12}=\hbar \Sigma_{21}$, which amounts to a choice of gauge that is related to our choice of $\langle\psi\rangle=\sqrt{n_{0}}$ being real.

Performing the Bogoliubov transformation to diagonalize the trial action 


$$
\begin{aligned}
S^{(\operatorname{tr})}\left[a^{*}, a\right]= & \sum_{\mathbf{k}, n}{ }^{\prime}\left(-i \hbar \omega_{n}+\epsilon_{\mathbf{k}}-\mu+\hbar \Sigma_{11}\right) a_{\mathbf{k}, n}^{*} a_{\mathbf{k}, n} \\
& +\frac{1}{2} \hbar \Sigma_{12} \sum_{\mathbf{k}, n}{ }^{\prime}\left(a_{\mathbf{k}, n}^{*} a_{-\mathbf{k},-n}^{*}+a_{\mathbf{k}, n} a_{-\mathbf{k},-n}\right),
\end{aligned}
$$

we find that the dispersion relation for the Bogoliubov quasiparticles is given by

$$
\hbar \Omega_{\mathbf{k}}=\sqrt{\left(\epsilon_{\mathbf{k}}-\mu^{\prime}\right)^{2}-\left(\hbar \Sigma_{12}\right)^{2}},
$$

where we have introduced $\mu^{\prime}=\mu-\hbar \Sigma_{11}$. Moreover, the usual coherence factors [32] for this transformation are given by $u_{\mathbf{k}}=\frac{1}{2}\left[\sqrt{\left(\epsilon_{\mathbf{k}}-\mu^{\prime}+\hbar \Sigma_{12}\right) / \hbar \Omega_{\mathbf{k}}}\right.$ $\left.+\sqrt{\hbar \Omega_{\mathbf{k}} /\left(\epsilon_{\mathbf{k}}-\mu^{\prime}+\hbar \Sigma_{12}\right)}\right] \quad$ and $\quad v_{\mathbf{k}}=\frac{1}{2}$ $\left[\sqrt{\left(\epsilon_{\mathbf{k}}-\mu^{\prime}+\hbar \Sigma_{12}\right) / \hbar \Omega_{\mathbf{k}}}-\sqrt{\hbar \Omega_{\mathbf{k}} /\left(\epsilon_{\mathbf{k}}-\mu^{\prime}+\hbar \Sigma_{12}\right)}\right]$, respectively. The Hugenholtz-Pines theorem [33] states that the exact normal and anomalous self-energies satisfy

$$
\mu=\hbar \Sigma_{11}(\mathbf{0}, 0)-\hbar \Sigma_{12}(\mathbf{0}, 0),
$$

leading to a gapless dispersion. This condition is, however, not imposed on our variational calculation since the
Hugenholtz-Pines relation is only valid in a stationary point of the action [12], and the variational approach by definition also describes nonstationary points. As we will see below, Eq. (9) will nonetheless turn out to hold for the threedimensional case if the temperatures are not too close to absolute zero. In two dimensions Eq. (9) will not be valid for temperatures unequal to zero, since it would lead to infrared diverging integrals in the thermodynamic potential, and is therefore excluded from the solution space by the variational principle. As mentioned above, this was actually our main motivation for also applying this approach to a twodimensional Bose gas.

The next step now is to calculate from the functional integral the thermodynamic potential $\Omega$, which equals

$$
\Omega=-\frac{1}{\beta} \ln Z_{\mathrm{tr}}+\frac{1}{\hbar \beta}\left\langle S^{(\mathrm{int})}\right\rangle_{\mathrm{tr}},
$$

where $S^{\text {(int) }}$ is the interaction term $S-S^{(\text {tr) }}$ found by a combination of Eqs. (6) and (7). Again using the abovementioned Bogoliubov transformation in combination with Wick's theorem [32], a straightforward calculation of this quantity leads to

$$
\begin{aligned}
\frac{1}{V} \Omega\left(\mu^{\prime}, \hbar \Sigma_{12}, n_{\mathbf{0}} ; \mu, T\right)= & \frac{1}{2} n_{\mathbf{0}}^{2} V_{\mathbf{0}}-\mu n+\frac{k_{B} T}{V} \sum_{\mathbf{k}}^{\prime} \ln \left(1-e^{\left.-\beta \hbar \Omega_{\mathbf{k}}\right)}+\frac{1}{2} \frac{1}{V} \sum_{\mathbf{k}}^{\prime}\left(\hbar \Omega_{\mathbf{k}}-\epsilon_{\mathbf{k}}+\mu^{\prime}\right)+\left(2 n_{\mathbf{0}} V_{\mathbf{0}}+\mu^{\prime}\right)\left(n-n_{\mathbf{0}}\right)\right. \\
& +\left(\hbar \Sigma_{12}-n_{\mathbf{0}} V_{\mathbf{0}}\right) \hbar \Sigma_{12} \alpha+V_{\mathbf{0}}\left(n-n_{\mathbf{0}}\right)^{2}+\frac{1}{2} \frac{1}{V} V_{\mathbf{0}}\left(\hbar \Sigma_{12}\right)^{2} \alpha^{2}
\end{aligned}
$$

where

$$
n=n_{\mathbf{0}}+\frac{1}{V} \sum_{\mathbf{k}, n}^{\prime}\left\langle a_{\mathbf{k}, n}^{*} a_{\mathbf{k}, n}\right\rangle=n_{\mathbf{0}}+\frac{1}{V} \sum_{\mathbf{k}}^{\prime}\left(\frac{\epsilon_{\mathbf{k}}-\mu^{\prime}}{\hbar \Omega_{\mathbf{k}}} N\left(\hbar \Omega_{\mathbf{k}}\right)+\frac{\epsilon_{\mathbf{k}}-\mu^{\prime}-\hbar \Omega_{\mathbf{k}}}{2 \hbar \Omega_{\mathbf{k}}}\right)
$$

is the equation for the density, and the dispersion $\hbar \Omega_{\mathbf{k}}$ is given by Eq. (8). Furthermore, we introduced the shorthand notation

$$
\alpha=\frac{1}{\hbar \Sigma_{12}} \frac{1}{V} \sum_{\mathbf{k}, n}^{\prime}\left\langle a_{\mathbf{k}, n}^{*} a_{-\mathbf{k},-n}^{*}\right\rangle=\frac{1}{V} \sum_{\mathbf{k}}{ }^{\prime} \frac{1+2 N\left(\hbar \Omega_{\mathbf{k}}\right)}{2 \hbar \Omega_{\mathbf{k}}}
$$

with $N(x)=1 /\left(e^{\beta x}-1\right)$ the Bose-Einstein distribution function, resulting from the sum over the Matsubara frequencies $\omega_{n}$. In particular, we have that $-\Sigma_{n} i / \beta\left(\hbar \omega_{n}-x\right)=N(x)$ [32].

Now, first of all, we have to minimize this expression for fixed $n_{\mathbf{0}}$, chemical potential $\mu$, and temperature $T$ with respect to the variational parameters $\hbar \Sigma_{12}$ and $\hbar \Sigma_{11}$, or, equivalently, $\hbar \Sigma_{12}$ and $\mu^{\prime}$. Putting $\left(\partial \Omega / \partial \hbar \Sigma_{12}\right)=0$ and $\left(\partial \Omega / \partial \mu^{\prime}\right)=0$ yields, respectively,

$$
\hbar \Sigma_{12}=n_{\mathbf{0}} \frac{V_{\mathbf{0}}}{1+V_{\mathbf{0}} \alpha}-\frac{2 n V_{\mathbf{0}}+\mu^{\prime}-\mu}{\left(1+V_{\mathbf{0}} \alpha\right)\left(\alpha+\hbar \Sigma_{12} \frac{\partial \alpha}{\partial \hbar \Sigma_{12}}\right)} \frac{\partial n}{\partial \hbar \Sigma_{12}}
$$

and

$$
\left(2 n V_{0}+\mu^{\prime}-\mu\right) \frac{\partial n}{\partial \mu^{\prime}}=\left[\hbar \Sigma_{12}\left(1+V_{0} \alpha\right)-n_{\mathbf{0}} V_{\mathbf{0}}\right] \hbar \Sigma_{12} \frac{\partial \alpha}{\partial \mu^{\prime}},
$$

which are both solved by

$$
\hbar \Sigma_{12}=n_{\mathbf{0}} \frac{V_{\mathbf{0}}}{1+V_{\mathbf{0}} \alpha}
$$

and

$$
\mu^{\prime}=\mu-2 n V_{\mathbf{0}} .
$$


Calculation of the second derivatives of the thermodynamic potential indeed shows that this solution corresponds to a minimum for any $V_{\mathbf{0}}>0$. For $V_{\mathbf{0}}<0$ the obtained solution corresponds to a maximum in the thermodynamic potential, implying an unstable solution. This is physically reasonable, since a negative $V_{0}$ implies an effective attraction between the constituent particles and thus a negative scattering length $a$. As a result, a magnetically trapped gas indeed turns out to be unstable against the formation of a dense (solid or liquid) phase in the (local density) limit, where the temperatures are much larger than the splittings $\hbar \omega$ of the one-particle eigenstates in the trap, and the correlation length is smaller than the typical trap size [34]. Quantitatively this means that $k_{B} T / \hbar \omega \gg l /|a| \gg 1$, where $l=\sqrt{\hbar / m \omega}$ is the extent of the lowest-lying unperturbed harmonic-oscillator state of the trap. However, in the opposite limit it has recently been shown by a numerical solution of the nonlinear Schrödinger equation that a condensate may be metastable for a sufficiently small occupation number [35]. Although this is a very interesting result in light of the fact that a number of alkali atoms, e.g., $\mathrm{Cs}$ and $\mathrm{Li}$, have negative scattering lengths $[36,37]$, we will not consider this inhomogeneous case here, and therefore restrict ourselves to $a>0$.

Starting with the second equation we immediately conclude that the solution for the normal self-energy is $\hbar \Sigma_{11}=2 n V_{\mathbf{0}}$, meaning that the variational calculation for this particular variable yields only the one-loop (i.e., HartreeFock) expression. The first equation shows however, that the anomalous self-energy $\hbar \Sigma_{12}$ is obtained in an approximation that goes far beyond one loop. In fact, as we will now show, it constitutes the many-body $T$-matrix approximation to the self-energy $\hbar \Sigma_{12}[12]$.

The many-body $T$ matrix $T^{\mathrm{MB}}\left(\mathbf{k}, \mathbf{k}^{\prime}, \mathbf{K} ; z\right)$, which is diagrammatically depicted in Fig. 1(a), describes the collision of two atoms having relative momenta $\mathbf{k}^{\prime}$ and $\mathbf{k}$ before and after the collision, and a total center-of-mass momentum $\mathbf{K}$ and center-of-mass energy $z$ in a medium containing the same atoms. The equation for the many-body $T$ matrix, which by construction sums all the ladder diagrams, can easily be derived if one realizes that the free propagation of the atoms is determined by the trial Hamiltonian, that can be diagonalized by a Bogoliubov transformation. A somewhat lengthy but straightforward calculation gives, in the thermodynamic limit,

$$
\begin{aligned}
T^{\mathrm{MB}}\left(\mathbf{k}, \mathbf{k}^{\prime}, \mathbf{K} ; z\right)= & V\left(\mathbf{k}-\mathbf{k}^{\prime}\right)+\int \frac{d \mathbf{k}^{\prime \prime}}{(2 \pi)^{d}} V\left(\mathbf{k}-\mathbf{k}^{\prime \prime}\right)\left\{\left[\frac{u_{+}^{2} u_{-}^{2}}{z-\hbar \Omega_{+}-\hbar \Omega_{-}}-\frac{v_{+}^{2} v_{-}^{2}}{z+\hbar \Omega_{+}+\hbar \Omega_{-}}\right]\left(1+N_{+}+N_{-}\right)\right. \\
& \left.+\left[\frac{u_{-}^{2} v_{+}^{2}}{z+\hbar \Omega_{+}-\hbar \Omega_{-}}-\frac{u_{+}^{2} v_{-}^{2}}{z-\hbar \Omega_{+}+\hbar \Omega_{-}}\right]\left(N_{+}-N_{-}\right)\right\} T^{\mathrm{MB}}\left(\mathbf{k}^{\prime \prime}, \mathbf{k}^{\prime}, \mathbf{K} ; z\right),
\end{aligned}
$$

where $N_{+} \equiv N\left(\hbar \Omega_{+}\right)$and $N_{-} \equiv N\left(\hbar \Omega_{-}\right)$. The plus sign denotes the momentum argument $(\mathbf{K} / 2)+\mathbf{k}^{\prime \prime}$, and similarly the minus sign denotes the argument $(\mathbf{K} / 2)-\mathbf{k}^{\prime \prime}$. This expression for the many-body $T$ matrix has a clear physical interpretation: Two incoming atoms with momenta $(\mathbf{K} / 2)+\mathbf{k}^{\prime \prime}$ and $(\mathbf{K} / 2)-\mathbf{k}^{\prime \prime}$ can scatter out of their momentum states either by the creation of two quasiparticles, by the creation of two quasiholes, and by the creation of one quasiparticle and one quasihole. The net rates for the first two processes are proportional to $u_{+}^{2} u_{-}^{2}\left[\left(1+N_{+}\right)\left(1+N_{-}\right)-N_{+} N_{-}\right]$and $v_{+}^{2} v_{-}^{2}\left[N_{+} N_{-}-\left(1+N_{+}\right)\left(1+N_{-}\right)\right]$, respectively, if we take the usual Bose enhancement factors for the scattering into occupied states into account. For the last process the quasiparticle can have either momentum $(\mathbf{K} / 2)+\mathbf{k}^{\prime \prime}$ or $(\mathbf{K} / 2)-\mathbf{k}^{\prime \prime}$. The net rates for these processes are proportional to $u_{-}^{2} v_{+}^{2}\left[\left(1+N_{-}\right) N_{+}-N_{-}\left(1+N_{+}\right)\right]$and $u_{+}^{2} v_{-}^{2}[(1$ $\left.\left.+N_{+}\right) N_{-}-N_{+}\left(1+N_{-}\right)\right]$, respectively. Together with the usual energy denominators, which are well known from second-order perturbation theory, this fully explains the structure of the right-hand side of Eq. (18).

Since we neglected all momentum dependencies of the self-energies $\hbar \Sigma_{11}$ and $\hbar \Sigma_{12}$ in the variational approach, we will also do so in the above equation for the $T$ matrix. When we furthermore set $u_{\mathbf{k}}=1$ and $v_{\mathbf{k}}=0$ by making use of the fact that the Bogoliubov spectrum $\hbar \Omega_{\mathbf{k}}$ deviates from $\epsilon_{\mathbf{k}}-\mu^{\prime}$ only for a very small momentum interval such that $\epsilon_{\mathbf{k}} \leq \hbar \Sigma_{12}<n V_{\mathbf{0}}$, we find that Eq. (18) reduces to

$$
\begin{aligned}
T^{\mathrm{MB}}(\mathbf{0}, \mathbf{0}, \mathbf{0} ; z)= & V_{\mathbf{0}}+V_{\mathbf{0}} T^{\mathrm{MB}}(\mathbf{0 , 0}, \mathbf{0} ; z) \\
& \times \int \frac{d \mathbf{k}}{(2 \pi)^{d}} \frac{1+2 N\left(\hbar \Omega_{\mathbf{k}}\right)}{z-2 \hbar \Omega_{\mathbf{k}}},
\end{aligned}
$$

with an error of $O\left(V_{0} n a \Lambda^{2}\right)$, which is negligible except in an extremely small interval of temperatures near absolute zero. By virtue of the fact that we neglected the momentum dependence of the scattering potential, this equation can easily be solved to give

$$
T^{\mathrm{MB}}(\mathbf{0 , 0 , 0} ; 0)=\frac{V_{\mathbf{0}}}{1+V_{\mathbf{0}} \alpha},
$$

which finally establishes our claim that the variational calculation gives the anomalous self-energy to within the $T$-matrix approximation, i.e., $\hbar \Sigma_{12}=n_{\mathbf{0}} T^{\mathrm{MB}}(\mathbf{0 , 0}, \mathbf{0} ; 0)$. Furthermore, Eq. (20) implies that $\alpha$ [cf. Eq. (13)] is equal to $1 / T^{\mathrm{MB}}(\mathbf{0}, \mathbf{0}, \mathbf{0} ; 0)-1 / V_{\mathbf{0}}$.

Note that in the calculation we are carrying out, this $T$-matrix still depends on the variational parameters $\hbar \Sigma_{11}$ and $\hbar \Sigma_{12}$ because they enter into the dispersion $\hbar \Omega_{\mathbf{k}}$ [cf. Eq. (8)]. Moreover, the many-body $T$ matrix can be related to the 

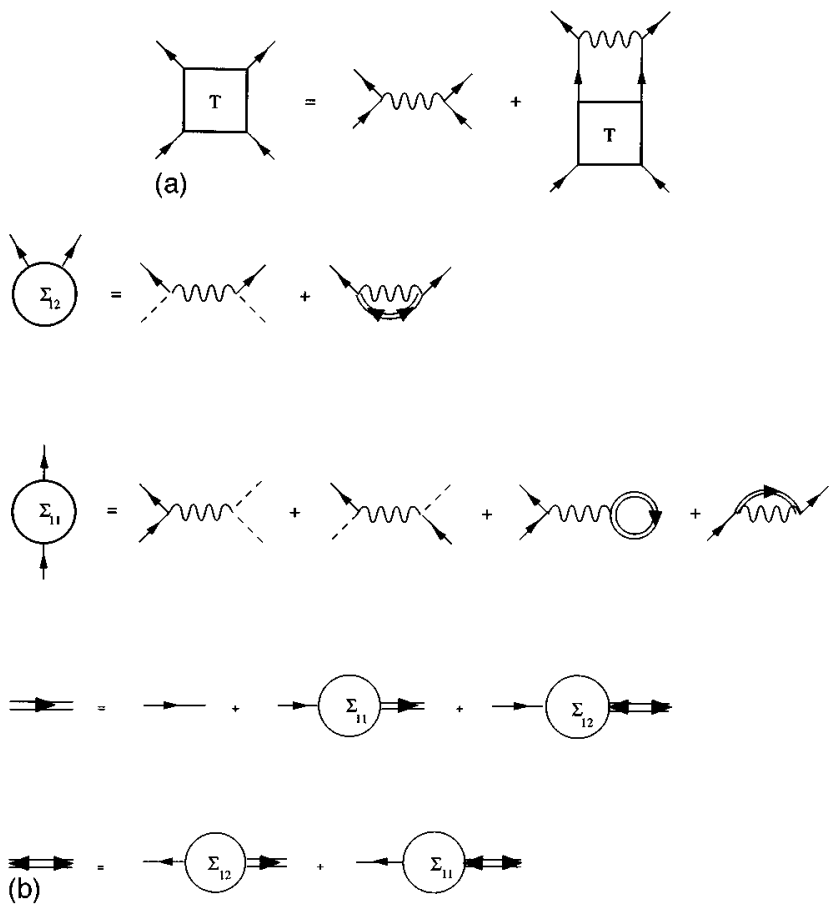

FIG. 1. Diagrammatic representations. (a) Diagrammatic representation of the $T$-matrix equation. (b) The normal and anomalous self-energies as found in the variational approach together with the expressions for the exact Green's functions. Note that iteration of the equation for $\Sigma_{12}$ generates the ladder diagrams. This is not the case for $\Sigma_{11}$.

two-body $T$ matrix, which describes the collision of two particles in a vacuum instead of a medium, through [12]

$$
\frac{1}{T^{\mathrm{MB}}(\mathbf{0}, \mathbf{0}, \mathbf{0} ; 0)}=\frac{1}{T^{2 B}\left(\mathbf{0}, \mathbf{0} ;-2 \mu^{\prime}\right)}+\frac{1}{V} \sum_{\mathbf{k}} \frac{N\left(\hbar \Omega_{\mathbf{k}}\right)}{\hbar \Omega_{\mathbf{k}}} .
$$

The low-energy limit ( $\epsilon_{\mathbf{k}} \ll \hbar^{2} / 2 m a^{2}$ ) of the two-body $T$ matrix has been studied extensively in the literature [38]. When the configuration space is three dimensional, it is accurately represented by

$$
T^{2 B}\left(\mathbf{0}, \mathbf{0} ; \epsilon_{\mathbf{k}}\right)=\frac{4 \pi a \hbar^{2}}{m},
$$

whereas

$$
T^{2 B}\left(\mathbf{0}, \mathbf{0} ; \boldsymbol{\epsilon}_{\mathbf{k}}\right)=\frac{4 \pi \hbar^{2} / m}{\pi i-\ln \left(k^{2} a^{2} / 8\right)-2 \gamma},
$$

when configuration space is two-dimensional. In these expressions $a$ denotes the scattering length or effective hardsphere radius of the interaction potential, and $\gamma \simeq 0.5772$ is Euler's constant. These long-wavelength expressions will enable us in a later stage to formulate our final results solely in terms of the scattering length instead of the full (microscopic) potential $V(\mathbf{x})$.

Comparing Eqs. (16) and (17), we conclude that the variational calculation as performed using the microscopic Hamiltonian of the Bose gas leads to an inconsistency: The normal self-energy is obtained to one-loop order only, whereas the anomalous self-energy is obtained in the $T$-matrix approximation, which contains any number of loops. The inconsistency can be better understood by diagrammatically analyzing the structure of Eqs. (16) and (17), making use of Eq. (12) for the density and the definition of $\alpha$ given in Eq. (13). The result is depicted in Fig. 1(b) together with the Dyson equation for the normal and anomalous Green's functions. It is clear from these diagrammatic equations that iteration will generate the ladder series for the anomalous self-energy $\hbar \Sigma_{12}$, but not for the normal self-energy $\hbar \Sigma_{11}$. Moreover, it shows that the set of diagrams which is being summed in our case of a complex $|\psi|^{4}$ theory gives at most the $T$ matrix. This is in contrast, for example, to the result obtained by Barnes and Ghandour [39]. They showed that a similar variational calculation for a real scalar $\phi^{4}$ theory yields selfenergies containing the ladder as well as the bubble diagrams.

To obtain consistent results with the above variational approach, we have to apply it to an effective Hamiltonian containing an effective interaction $V_{\mathbf{0}}^{\text {eff }}$. This procedure is often followed in the literature with $V_{\mathbf{0}}^{\text {eff }}$ equal to $4 \pi a \hbar^{2} / \mathrm{m}$. It was introduced by Huang and Yang in the 1950s [40], and has since been known as the pseudopotential method. Using the effective Hamiltonian implies that we can replace $V_{0}$ everywhere by the effective potential $V_{\mathbf{0}}^{\text {eff }}$, except in the condensate contribution $n_{\mathbf{0}}^{2} V_{\mathbf{0}} / 2$, for reasons that will become clear shortly, but are also known from the work of Huang and Yang. Again, performing the minimization procedure now leads to $\hbar \Sigma_{12}=n_{\mathbf{0}} V_{\mathbf{0}}^{\text {eff }} /\left(1+V_{\mathbf{0}}^{\text {eff }} \alpha\right)$ and $\mu^{\prime}=\mu-2 n V_{\mathbf{0}}^{\text {eff }}$, where $\alpha=1 / T^{\mathrm{MB}}(\mathbf{0}, \mathbf{0}, \mathbf{0} ; 0)-1 / V_{\mathbf{0}}^{\text {eff }}[$ cf. Eqs. (16) and (17)]. It is readily seen that taking $V_{\mathbf{0}}^{\mathrm{eff}}$ equal to the many-body $T$ matrix evaluated at the minimum of $\Omega$ that is located at the point in the $\mu^{\prime}-\hbar \Sigma_{12}$ plane given by the above solutions, indeed leads to the desired self-energies

$$
\begin{aligned}
& \hbar \Sigma_{12}=n_{\mathbf{0}} T^{\mathrm{MB}}(\mathbf{0 , 0}, \mathbf{0} ; 0), \\
& \hbar \Sigma_{11}=2 n T^{\mathrm{MB}}(\mathbf{0 , 0}, \mathbf{0} ; 0)
\end{aligned}
$$

in the $T$-matrix approximation. Thus we see that the effective Hamiltonian needed to obtain consistent results contains the many-body $T$ matrix as the effective interparticle interaction, instead of the two-body $T$ matrix that is used in the pseudopotential method.

The thermodynamic potential at this minimum in the first instance equals

$$
\begin{aligned}
\frac{1}{V} \Omega\left(n_{\mathbf{0}} ; \mu, T\right)= & \frac{1}{2} n_{\mathbf{0}}^{2} V_{\mathbf{0}}-\mu n+\frac{k_{B} T}{V} \sum_{\mathbf{k}} \ln \left(1-e^{\left.-\beta \hbar \Omega_{\mathbf{k}}\right)}\right. \\
& +\frac{1}{2} \frac{1}{V} \sum_{\mathbf{k}}^{\prime}\left(\hbar \Omega_{\mathbf{k}}-\epsilon_{\mathbf{k}}+\mu^{\prime}\right) \\
& +\left(2 n_{\mathbf{0}} T^{\mathrm{MB}}(\mathbf{0}, \mathbf{0}, \mathbf{0} ; 0)+\mu^{\prime}\right)\left(n-n_{\mathbf{0}}\right) \\
& +T^{\mathrm{MB}}(\mathbf{0}, \mathbf{0}, \mathbf{0} ; 0)\left(n-n_{\mathbf{0}}\right)^{2}
\end{aligned}
$$

from which we still have to eliminate the $V_{\mathbf{0}}$ dependence occurring in the first term, as well as the ultraviolet divergence in the fourth term of the right-hand side. As is well 
known, these two matters are intimately connected, and can both be resolved by writing the sum of these two terms as

$$
\begin{aligned}
& \frac{1}{2} n_{\mathbf{0}}^{2} V_{\mathbf{0}}+\frac{1}{2} \frac{1}{V} \sum_{\mathbf{k}}{ }^{\prime}\left(\hbar \Omega_{\mathbf{k}}-\epsilon_{\mathbf{k}}+\mu^{\prime}+\frac{\left(\hbar \Sigma_{12}\right)^{2}}{2 \hbar \Omega_{\mathbf{k}}}\right) \\
& -\frac{1}{2}\left(\hbar \Sigma_{12}\right)^{2} \frac{1}{V} \sum_{\mathbf{k}}{ }^{\prime} \frac{1}{2 \hbar \Omega_{\mathbf{k}}} .
\end{aligned}
$$

By also adding and subtracting $\left(\hbar \Sigma_{12}\right)^{2}(1 / V) \Sigma_{\mathbf{k}} N\left(\hbar \Omega_{\mathbf{k}}\right) /$ $2 \hbar \Omega_{\mathbf{k}}$ and realizing that $\hbar \Sigma_{12}=n_{\mathbf{0}} T^{\mathrm{MB}}(\mathbf{0 , 0}, \mathbf{0} ; 0)$, we see that
$V_{0}$ is exactly renormalized to the many-body $T$ matrix if one of the $T^{\mathrm{MB}}$ 's in $\left(\hbar \Sigma_{12}\right)^{2}$ is replaced by $V_{\mathbf{0}}$, since then

$$
\begin{aligned}
& \frac{1}{2} n_{\mathbf{0}}^{2} V_{\mathbf{0}}-\frac{1}{2} n_{\mathbf{0}}^{2} V_{\mathbf{0}} T^{\mathrm{MB}}(\mathbf{0 , 0 , 0} ; 0) \frac{1}{V} \sum_{\mathbf{k}}{ }^{\prime} \frac{1+2 N\left(\hbar \Omega_{\mathbf{k}}\right)}{2 \hbar \Omega_{\mathbf{k}}} \\
& =\frac{1}{2} n_{\mathbf{0}}^{2} T^{\mathrm{MB}}(\mathbf{0}, \mathbf{0}, \mathbf{0} ; 0)
\end{aligned}
$$

due to Eq. (19). The final expression for the thermodynamic potential thus becomes

$$
\begin{aligned}
& \frac{1}{V} \Omega\left(n_{\mathbf{0}} ; \mu, T\right)=\frac{1}{2} n_{\mathbf{0}}^{2} T^{\mathrm{MB}}(\mathbf{0}, \mathbf{0}, \mathbf{0} ; 0)-\mu n+\frac{k_{B} T}{V} \sum_{\mathbf{k}} \ln \left(1-e^{-\beta \hbar \Omega_{\mathbf{k}}}\right)+\frac{1}{2} \frac{1}{V} \sum_{\mathbf{k}}{ }^{\prime}\left(\hbar \Omega_{\mathbf{k}}-\epsilon_{\mathbf{k}}+\mu^{\prime}+\frac{\left(\hbar \Sigma_{12}\right)^{2}}{2 \hbar \Omega_{\mathbf{k}}}\right) \\
& +\frac{1}{2}\left(\hbar \Sigma_{12}\right)^{2} \frac{1}{V} \sum_{\mathbf{k}}{ }^{\prime} \frac{N\left(\hbar \Omega_{\mathbf{k}}\right)}{\hbar \Omega_{\mathbf{k}}}+\left(2 n_{\mathbf{0}} T^{\mathrm{MB}}(\mathbf{0}, \mathbf{0}, \mathbf{0} ; 0)+\mu^{\prime}\right)\left(n-n_{\mathbf{0}}\right)+T^{\mathrm{MB}}(\mathbf{0 , 0 , 0} ; 0)\left(n-n_{\mathbf{0}}\right)^{2},
\end{aligned}
$$

with $\hbar \Sigma_{12}$ given by Eq. (24) and $\mu^{\prime}=\mu$ $-2 n T^{\mathrm{MB}}(\mathbf{0 , 0}, \mathbf{0} ; 0)$. Note that this derivation by no means depends on the validity or violation of the Hugenholtz-Pines relation, and that we now have obtained the thermodynamic potential containing only the many-body $T$ matrix and not the interaction parameter $V_{\mathbf{0}}$. The fourth term on the righthand side can be shown to be at most of $O\left(\sqrt{n a^{3}}\right)$ in three dimensions, and of $O\left(1 / \ln \left(1 / n a^{2}\right)\right)$ in two dimensions, and will henceforth be neglected.

Equation (28) is the central result of this section, and requires only the use of Bogoliubov wave functionals with momentum- and energy-independent self-energies. Although obtained by a variational ansatz for the wave functionals and an appropriate effective Hamiltonian, it has lead to a correct expression for the thermodynamic potential in the $T$-matrix approximation. As a proof of this fact we will now in an independent way derive Eq. (28) directly from the original action $S\left[a^{*}, a\right]$, for which in Eq. (5) we have written down the quadratic part, avoiding the use of a variational approach. For this purpose, we also treat the off-diagonal part of $S^{(2)}$ as a perturbation, and consider only the diagonal part as the unperturbed action. Thus $\left\langle a_{\mathbf{k}, n}^{*} a_{\mathbf{k}, n}\right\rangle=-\hbar /\left(i \hbar \omega_{n}-\epsilon_{\mathbf{k}}+\mu^{\prime}\right)$. In the first instance we then immediately write

$$
\begin{aligned}
\frac{1}{V} \Omega= & \frac{1}{2} n_{\mathbf{0}}^{2} V_{\mathbf{0}}-\mu n_{\mathbf{0}}+\frac{k_{B} T}{V} \sum_{\mathbf{k}}{ }^{\prime} \ln \left(1-e^{-\beta\left(\epsilon_{\mathbf{k}}-\mu^{\prime}\right)}\right) \\
& -T^{\mathrm{MB}}(\mathbf{0}, \mathbf{0}, \mathbf{0} ; 0)\left(n-n_{\mathbf{0}}\right)^{2},
\end{aligned}
$$

where the last term compensates for the double counting of the interactions. The first term can now be improved to the $T$-matrix level by performing perturbation theory in the offdiagonal parts of the action $S^{(2)}$. The lowest-order term contributes $-\left(n_{\mathbf{0}} V_{\mathbf{0}}\right)^{2}(1 / V) \Sigma_{\mathbf{k}}\left(1+2 N\left(\epsilon_{\mathbf{k}}-\mu^{\prime}\right)\right) / 4\left(\epsilon_{\mathbf{k}}-\mu^{\prime}\right)$ to the thermodynamic potential, whereas the consecutive terms are of $O\left(n_{0} a^{3}\right)$ or $O\left(1 / \ln \left(1 / n_{0} a^{2}\right)\right)$ in three and two dimensions, respectively, and negligible. Combining this lowestorder term with $n_{\mathbf{0}}^{2} V_{\mathbf{0}} / 2$, the thermodynamic potential now reads

$$
\begin{aligned}
\frac{1}{V} \Omega= & \frac{1}{2} n_{\mathbf{0}}^{2} T^{\mathrm{MB}}(\mathbf{0}, \mathbf{0}, \mathbf{0} ; 0)-\mu n_{\mathbf{0}} \\
& +\frac{k_{B} T}{V} \sum_{\mathbf{k}}{ }^{\prime} \ln \left(1-e^{-\beta\left(\epsilon_{\mathbf{k}}-\mu^{\prime}\right)}\right) \\
& -T^{\mathrm{MB}}(\mathbf{0 , 0}, \mathbf{0} ; 0)\left(n-n_{\mathbf{0}}\right)^{2} .
\end{aligned}
$$

This result now has to be compared with Eq. (28). First, we have to show, lest they be equal, that

$$
\begin{aligned}
& \frac{k_{B} T}{V} \sum_{\mathbf{k}}{ }^{\prime} \ln \left(1-e^{\left.-\beta \hbar \Omega_{\mathbf{k}}\right)+\frac{1}{2}\left(\hbar \Sigma_{12}\right)^{2}} \frac{1}{V} \sum_{\mathbf{k}}{ }^{\prime} \frac{N\left(\hbar \Omega_{\mathbf{k}}\right)}{\hbar \Omega_{\mathbf{k}}}\right. \\
& \quad=\frac{k_{B} T}{V} \sum_{\mathbf{k}}{ }^{\prime} \ln \left(1-e^{-\beta\left(\epsilon_{\mathbf{k}}-\mu^{\prime}\right)}\right) .
\end{aligned}
$$

That this equality holds to a very good approximation can be demonstrated simply by performing an expansion of $\hbar \Omega_{\mathbf{k}}$, which leads to

$$
\begin{aligned}
\ln \left(1-e^{-\beta \hbar \Omega_{\mathbf{k}}}\right)= & \ln \left(1-e^{-\beta\left(\epsilon_{\mathbf{k}}-\mu^{\prime}\right)}\right) \\
& -\beta \frac{\left(\hbar \Sigma_{12}\right)^{2}}{2\left(\epsilon_{\mathbf{k}}-\mu^{\prime}\right)} N\left(\epsilon_{\mathbf{k}}-\mu^{\prime}\right)+\text { h.o.t. }
\end{aligned}
$$

(h.o.t. stands for higher-order terms) where the ratio of the higher-order terms and the first-order term in the expansion is either of $O\left(n_{0} a \Lambda^{2}\right)$ or of $O\left[\left(n_{0} \Lambda^{2} / \ln \left(1 / n_{0} a^{2}\right)\right)\right]$ in the regime where these parameters are small. Close to zero temperature these parameters become large, but what has to be compared in that situation is the contribution of these terms to the total thermodynamic potential. Due to the Bose factors their contribution becomes negligible. Second, the two respective terms $\left(n-n_{\mathbf{0}}\right)^{2}$ in Eqs. (28) and (30) also differ due to the difference in dispersion. Again, expanding $\hbar \Omega_{\mathbf{k}}$, one can show that the error here is of the same order as above, 


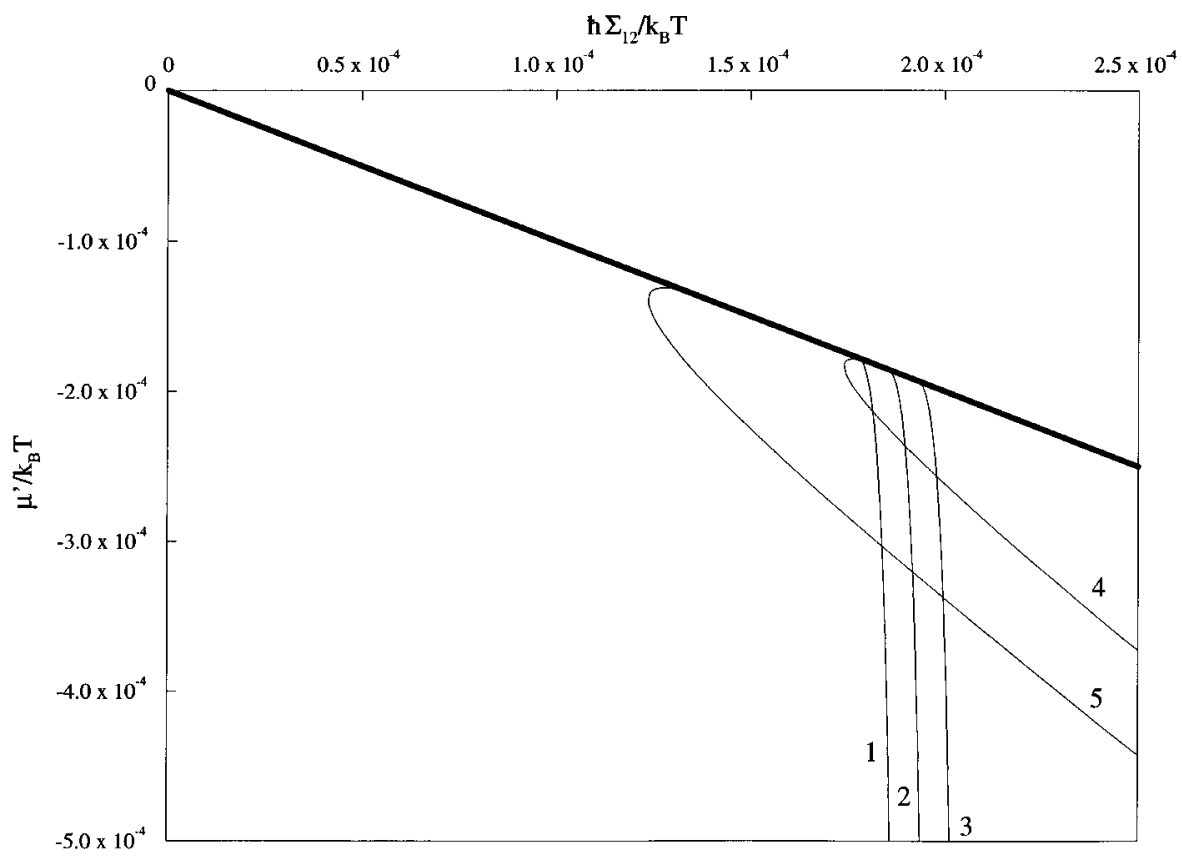

FIG. 2. Lines of constant chemical potential (4 and 5 , the first having the largest chemical potential), and solution curves of the equation $\hbar \Sigma_{12}=n_{0} T^{\mathrm{MB}}$ $(\mathbf{0 , 0}, \mathbf{0} ; 0)$ in the $\mu^{\prime}-\hbar \Sigma_{12}$ plane for (1) $n_{0} a^{3}=4.8 \times 10^{-12}$, (2) $n_{0} a^{3}=5.0 \times 10^{-12}$, and (3) $\quad n_{0} a^{3}=5.2 \times 10^{-12}$. Also shown is the line on which the Hugenholtz-Pines relation is satisfied.

and is negligible. Thus, as this independent calculation shows, Eq. (28) obtained from the modified variational calculation is indeed the correct result for the thermodynamic potential.

The above argument shows that the results obtained in Eqs. (24), (25), and (28) can also be interpreted in a different manner. First we break the symmetry of the theory by hand. We subsequently calculate the normal and anomalous selfenergies $\hbar \Sigma_{11}$ and $\hbar \Sigma_{12}$ in the $T$-matrix approximation. This straightforwardly gives Eqs. (24) and (25) (see also Ref. 12). We then also calculate the thermodynamic potential in the $T$-matrix approximation, yielding, as we have seen, Eq. (28). Thus we again arrive without reference at a variational calculation at the same set of equations and in particular at the same thermodynamic potential as a function of condensate density, chemical potential and temperature. The value of the variational calculation lies therein, in that it gives a clear picture of the physics involved in the microscopic calculation, and in particular shows what the correct, i.e., selfconsistent, effective Hamiltonian of the gas is.

The final step we now have to take is to find the value of $n_{0}$ which minimizes the thermodynamic potential at given $\mu$ and $T$. Because this program has to be implemented numerically, we now restrict ourselves to the specific example of spin-polarized hydrogen atoms in both three-dimensional (Sec. III A) and two-dimensional (Sec. III B) configurations. In the case of the system being three dimensional, this leads to improvements on existing approaches to this problem, and we therefore particularly present results for the condensate fraction and the $p-n^{-1}$ diagram.

The equations which are relevant for a numerical implementation are first of all $\hbar \Sigma_{12}=n_{\mathbf{0}} T^{\mathrm{MB}}(\mathbf{0 , 0 , 0} ; 0)$ and $\mu^{\prime}=\mu-2 n T^{\mathrm{MB}}(\mathbf{0 , 0 , 0} ; 0)$ [cf. Eqs. (24) and (25)], localizing the minimum of the thermodynamic potential. To solve them for various values of $n_{\mathbf{0}}$, we first locate the $\left(\hbar \Sigma_{12}, \mu^{\prime}\right)$ combinations for which the first equation is satisfied. Next, we locate $\left(\hbar \Sigma_{12}, \mu^{\prime}\right)$ combinations which yield the same chemical potential $\mu$ by means of the second equation. For each value of $n_{0}$ these two curves have a point of intersection, which thus solves both equations. The value of $\Omega$ as a function of $n_{0}$ for fixed $\mu$ and $T$ is then obtained by means of Eq. (28). Repeating the above procedure several times, we determine the value of $n_{\mathbf{0}}$, minimizing the thermodynamic potential. After this the properties of the system are fully determined, and it is possible to calculate, e.g., the condensate fraction and the superfluid density as a function of temperature.

\section{SPIN-POLARIZED ATOMIC HYDROGEN}

In order to obtain Bose-Einstein condensation, one magnetically traps hydrogen atoms in the doubly polarized $|d\rangle=|\uparrow \uparrow\rangle$ state of the upper hyperfine manifold, resulting in a well-known effectively repulsive triplet interaction between the atoms with a value of the scattering length of $1.34 a_{0}$ [41]. Typical sample densities are $10^{13}-10^{14}$ atoms per cubic centimeter, with a corresponding critical temperature on the order of tens of $\mathrm{mK}$.

For the two-dimensional system one uses atoms in the doubly polarized $|b\rangle=|\downarrow \downarrow\rangle$ state of the lower hyperfine manifold [2] adsorbed on a superfluid helium film. The effective interaction between the adsorbed atoms is in a very good approximation given by the weighed average of the volume triplet potential, using the square of the bound-state wave function pertaining to the motion perpendicular to the surface, as a weighting factor [42]. This procedure leads to a two-dimensional scattering length with a value of $2.40 a_{0}$ [38]. The densities one aims at in this experimental setup are typically some $10^{13}$ atoms per square centimeter, which corresponds to a critical temperature in the range of 50-100 $\mathrm{mK}$.

\section{A. Three-dimensional case}

In Fig. 2 we show, for three different values of $n_{0} a^{3}$, the curves in the $\hbar \Sigma_{12}-\mu^{\prime}$ plane on which the equality 


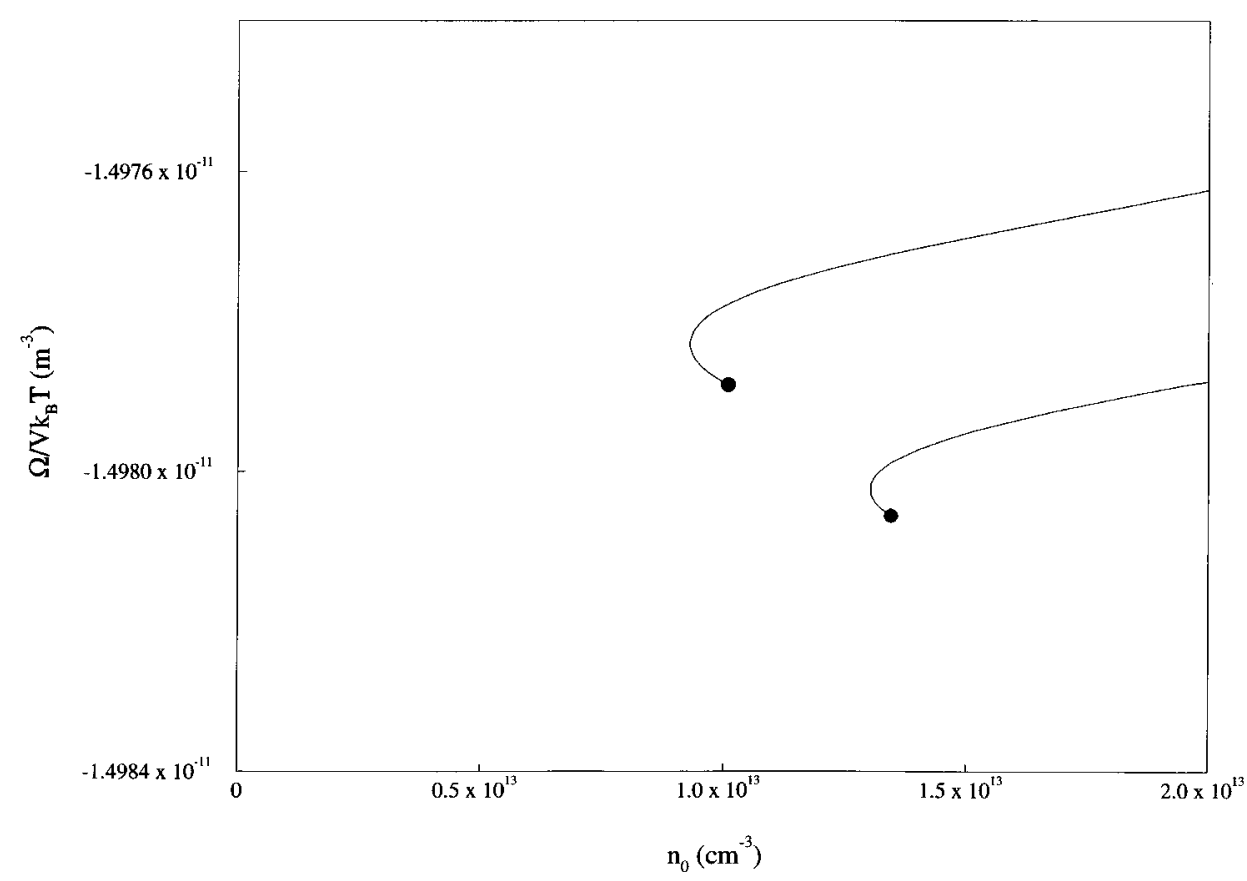

FIG. 3. Thermodynamic potential vs condensate density for the same two values of the chemical potential as in Fig. 2 and at a temperature of $30 \mu \mathrm{K}$. The dots indicate the starting points on the Hugenholtz-Pines line of the corresponding curves in the $\mu^{\prime}$ $\hbar \Sigma_{12}$ plane. The graph shows that at the minimum the HugenholtzPines condition is indeed satisfied.

$\hbar \Sigma_{12}=n_{\mathbf{0}} T^{\mathrm{MB}}(\mathbf{0}, \mathbf{0}, \mathbf{0} ; 0)$ holds, as well as two curves on which the chemical potential $\mu$ is constant, both at a temperature of $30 \mu \mathrm{K}$. The straight line $\mu^{\prime}=-\hbar \Sigma_{12}$ corresponds to the conditions where the Hugenholtz-Pines theorem is satisfied. The area $\hbar \Sigma_{12}>\left|\mu^{\prime}\right|$ is of course not part of the solution space since it would imply a complex (unstable) dispersion relation. By calculating the thermodynamic potential at the intersections of the lines, we infer the relation between $\Omega$ and the condensate density $n_{\mathbf{0}}$ for a given chemical potential.

This is shown in Fig. 3 for the same two values of the chemical potential. From this plot we find that the $n_{\mathbf{0}}$ minimizing the thermodynamic potential for given $\mu$ indeed corresponds to the situation where the Hugenholtz-Pines theorem is satisfied, i.e., $\mu^{\prime}=-\hbar \Sigma_{12}$. Note that this is a result of the minimization procedure, and is not assumed beforehand. The Hugenholtz-Pines relation, however, turns out not to hold in every situation. For extreme conditions, very close to zero temperature, the position of the minimum is shifted slightly from the Hugenholtz-Pines line (see also Fig. 12 in Sec. III B). At $T=0$ one can, starting from Eq. (28) with general $\hbar \Sigma_{12}$ and $\mu^{\prime}$, also prove analytically that this is the case. We can, however, understand this shortcoming as follows. The deviation from the Hugenholtz-Pines condition is due to an overestimate of the effective interaction between the atoms. Indeed, a smaller value of the $T$ matrix, together with a larger value of the condensate density to ensure that the chemical potential $\mu$ remains the same, shifts the minimum toward the Hugenholtz-Pines line. This overestimate of the effective interaction is due to the neglect of the influence of the coherence factors in the expression for the many-body $T$ matrix in Eq. (19), i.e., due to putting $u_{\mathbf{k}}=1$ and $v_{\mathbf{k}}=0$. Taking these coherence factors into account will cause a decrease of the $T$ matrix, which presumably solves the problem that the minimum of the $\Omega-n_{0}$ curve does not coincide with the Hugenholtz-Pines criterion. The region where the neglect of the coherence factors becomes important corresponds to the condition $n a \Lambda^{2} \gtrsim 1$, which is indeed in the extremely low-temperature range where our numerical results show a minimum in the thermodynamic potential that is slightly shifted from the Hugenholtz-Pines line. Since this temperature range will be very difficult to obtain experimentally, we will not consider it further here.

Outside this range, all the properties of the system can now be calculated accurately. In Fig. 4 we plot the condensate fraction as a function of temperature for a density of $10^{14} \mathrm{~cm}^{-3}$. The inset shows the behavior near the critical temperature following from our calculation, as well as from a calculation in which we have replaced the many-body $T$ matrix by the two-body $T$ matrix. The latter is essentially what has been done in earlier approaches to the dilute Bose gas [6]. Using the two-body $T$ matrix, however, produces a double valuedness in this curve, leading inevitably to the conclusion that there is a first-order phase transition. This double valuedness also shows up in the equation of state depicted in Fig. 5. Again, the equation of state is single valued if the many-body $T$ matrix is used. Our variational approach has therefore succesfully resolved the long-standing problem concerning the double valuedness in the equation of state. It shows that it is essential to include the effect of the surrounding medium on the collision of two hydrogen atoms, which leads effectively to a temperature-dependent scattering amplitude and a softening of the dispersion near the critical temperature. We calculated the effect of the medium on the effective scattering length explicitly for the above situation where $n=10^{14} \mathrm{~cm}^{-3}$, and show in Fig. 6 the ratio of the many-body $T$ matrix to the two-body $T$ matrix as a function of temperature. The fact that the many-body $T$ matrix, and therefore the effective interaction of the atoms, goes to zero at the critical temperature, is correct when neglecting momentum and frequency dependencies, as can be shown from an exact renormalization-group equation for the four-point vertex function [43].

However, the many-body $T$ matrix $T^{\mathrm{MB}}\left(\mathbf{k}, \mathbf{k}^{\prime}, \mathbf{K} ; z\right)$ does not go to zero for nonzero momenta and frequencies. This explains the feature seen in the inset of Fig. 7, where we plot 


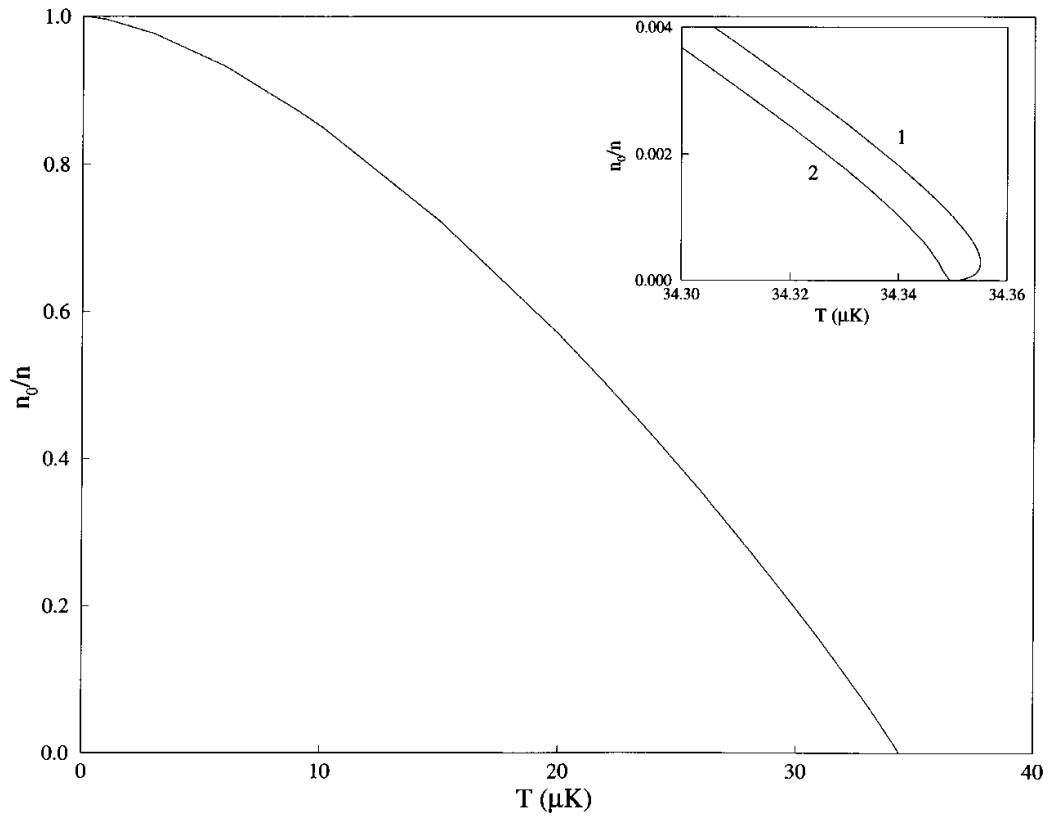

FIG. 4. The condensate fraction as a function of temperature for a total density of $10^{14} \mathrm{~cm}^{-3}$. The inset shows the behavior of the condensate fraction near the critical temperature revealing the double valuedness (curve 1) if one uses the two-body $T$ matrix, which is resolved if one uses the many-body $T$ matrix that includes the effect of the medium on the collision (curve 2). the $p-n^{-1}$ diagram resulting from our calculations. Near the critical density the curve shows a van der Waals loop, which, by means of a Maxwell construction, would still imply a first-order phase transition. This behavior is due to the fact that the thermodynamic potential Eq. (28), and hence the pressure $p=-\Omega / V$, always contains the many-body $T$ matrix evaluated at zero momenta and energies. Therefore, the van der Waals loop shown in Fig. 7 is solely a consequence of the behavior of the ratio $T^{\mathrm{MB}}(\mathbf{0 , 0}, \mathbf{0} ; 0) / T^{2 B}(\mathbf{0 , 0} ; 0)$ near the critical temperature (see Fig. 6). It would not have been present had we used a more accurate expression for the thermodynamic potential, which in principle contains integrals over the many-body $T$ matrix evaluated at finite momenta and energies. Indeed, as was shown in a separate paper using different methods [44], the term $T^{\mathrm{MB}}(\mathbf{0 , 0}, \mathbf{0} ; 0)\left(n-n_{\mathbf{0}}\right)^{2}$ should be replaced by $T^{2 B}(\mathbf{0 , 0} ; 0)\left(n-n_{\mathbf{0}}\right)^{2}$, which removes the van der Waals loop from the $p-n^{-1}$ diagram. Hence the first-order phase transition implied from Fig. 7 is an artifact of our long-wavelength approximation, which breaks down near the critical temperature. We stress, however, that the softening of the dispersion discussed earlier, resolving the problems related to the double valuedness in the equation of state near the critical temperature, in contrast, is not an artifact because the dispersion relation does contain $T^{\mathrm{MB}}(\mathbf{0 , 0 , 0} 0)$ in the long-wavelength limit. This concludes our discussion of the three-dimensional system, and we now turn to the two-dimensional case.

\section{B. Two-dimensional case}

In Fig. 8 we show the solution curves of $\hbar \Sigma_{12}$ $=n_{\mathbf{0}} T^{\mathrm{MB}}(\mathbf{0 , 0 , 0} ; 0)$ for three different values of $n_{\mathbf{0}} a^{2}$ at a

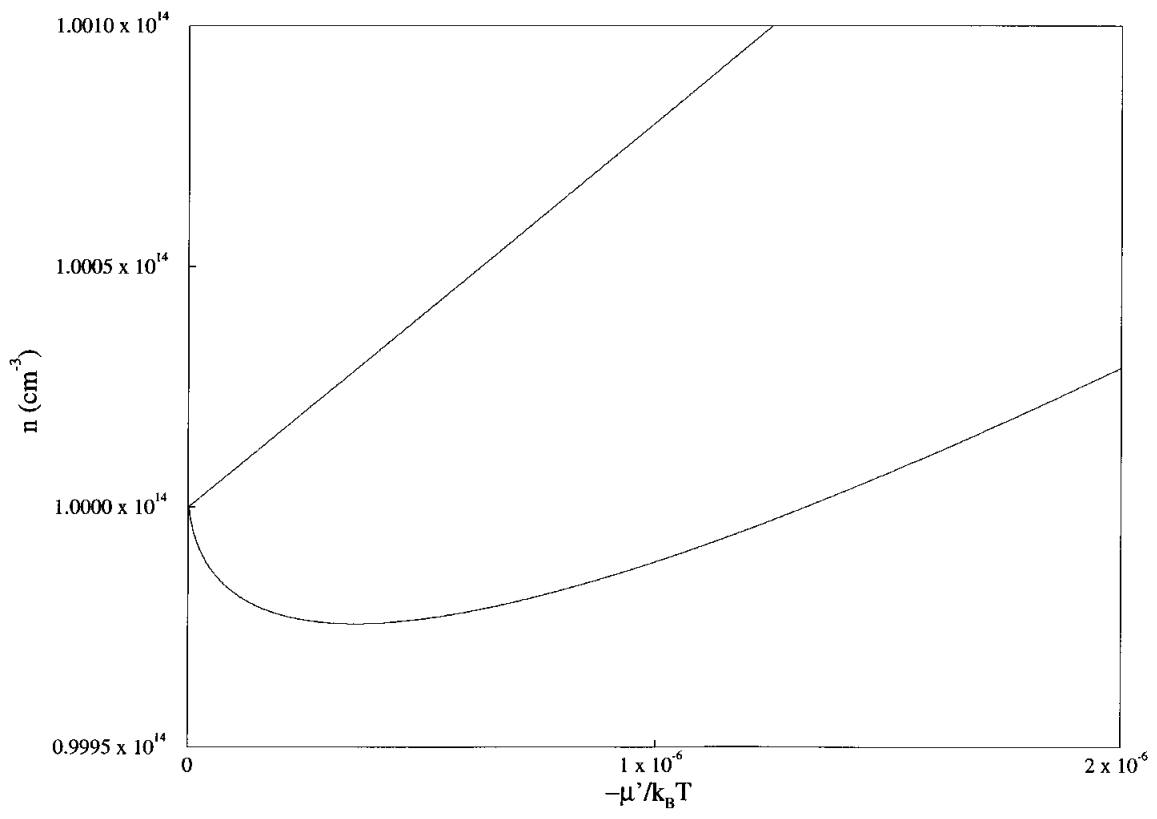

FIG. 5. The equation of state for small $\mu^{\prime}$ at the critical temperature for a system with $n=10^{14} \mathrm{~cm}^{-3}$, showing the double valuedness also discussed in Fig. 4. 


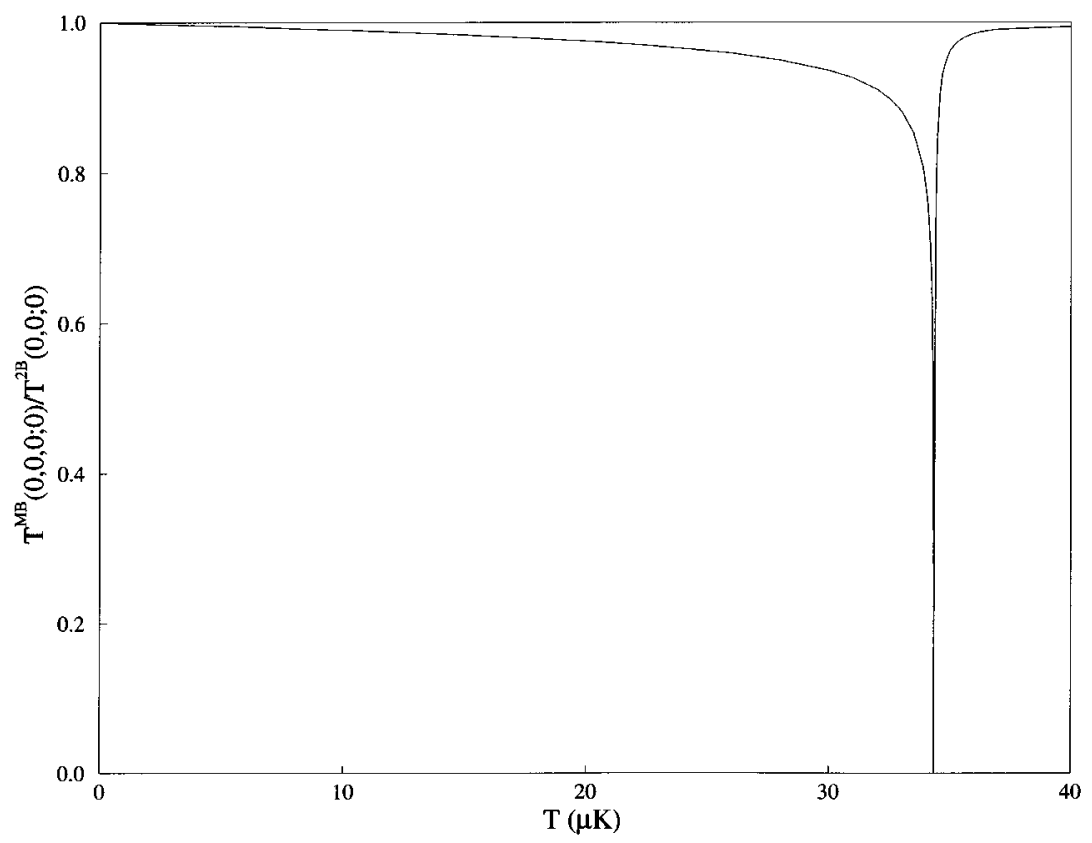

FIG. 6. The ratio of the many-body $T$ matrix to the two-body $T$ matrix at a density of $10^{14}$ $\mathrm{cm}^{-3}$, showing that $T^{\mathrm{MB}}(\mathbf{0}, \mathbf{0}, \mathbf{0} ; 0)$ goes to zero at the critical temperature.

temperature of $50 \mathrm{mK}$, as well as two curves on which the chemical potential $\mu$ is constant. In addition to the area $\hbar \Sigma_{12}>\left|\mu^{\prime}\right|$, the Hugenholtz-Pines line itself is also not part of the solution space, since it would imply a gapless dispersion and therefore infrared divergences. This is also the cause of the essential difference between Figs. 8 and 2. To avoid infrared divergences in the first case, the solution curves of $\hbar \Sigma_{12}=n_{\mathbf{0}} T^{\mathrm{MB}}(\mathbf{0 , 0}, \mathbf{0} ; 0)$ are forced to start in the origin of the $\mu^{\prime}-\hbar \Sigma_{12}$ plane, in contrast to what happens in three dimensions (cf. Fig. 2). This is a crucial point of difference, and results in the fact that the curves of the thermodynamic potential versus chemical potential all start in one and the same point, corresponding to $\mu=\hbar \Sigma_{12}=\mu^{\prime}=0$, as shown in Fig. 9, where $n_{\mathbf{0}}$ increases going from curve 1 to curve 4.
From this graph the following is then immediately clear: For $\mu<0$ the value of $n_{0}$ yielding the lowest thermodynamic potential is zero, whereas for $\mu>0$ the value of $n_{0}$ has to be infinite to give the lowest value of $\Omega$. Thus for $\mu>0$ we cannot fix the number of particles in the system to a finite value, and we conclude that the variational approach signals the onset of an instability at $\mu=0$, but cannot describe the phase below the critical temperature. Above $T_{c}$ it describes the two-dimensional Bose gas in the ladder approximation. What we can do is to deduce from the condition $\mu=0$ the relation between the critical temperature and density as it follows from our variational approach. This is shown in Fig. 10 , where we plot $n_{c}$ versus $T_{c}$. As expected, we find that the critical temperature is overestimated, and $n_{c} \Lambda_{c}^{2}$ is smaller than 4. This is consistent with the fact that the variational

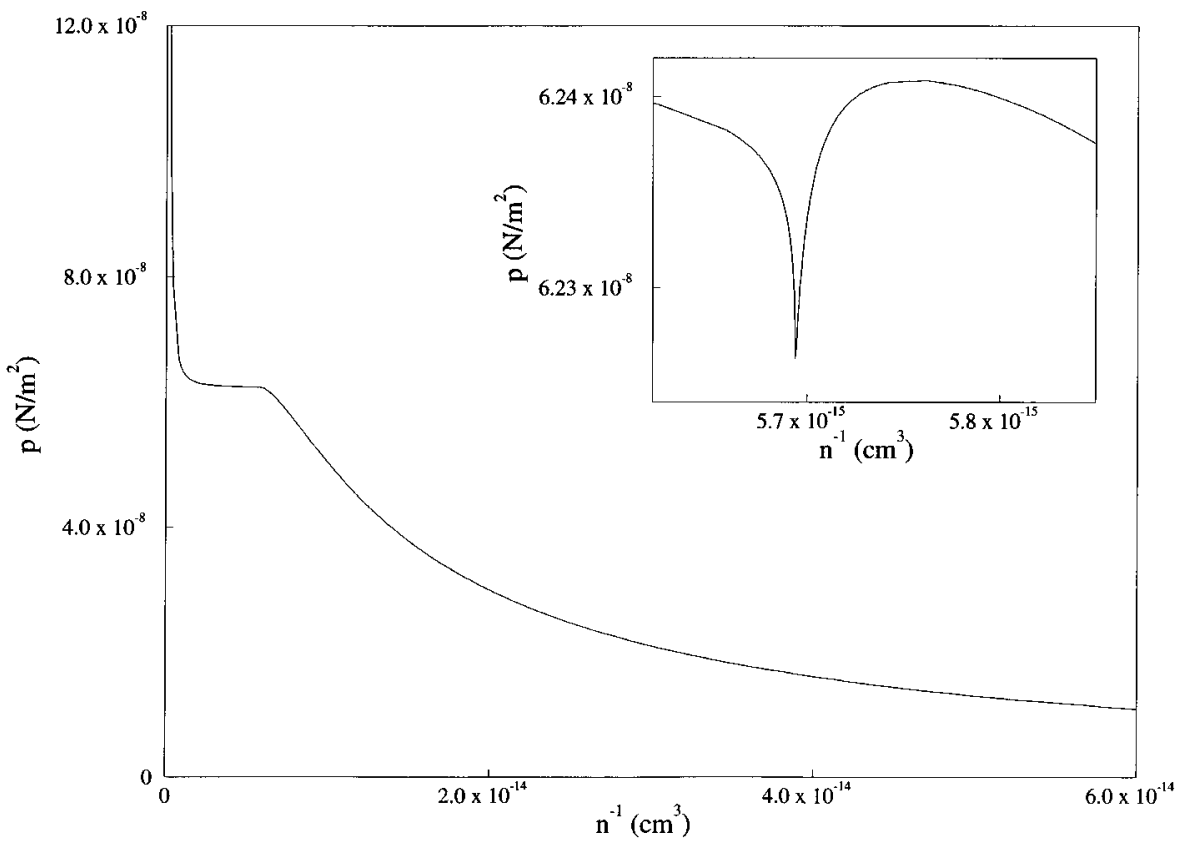

FIG. 7. Pressure vs inverse density at $T=50 \mu \mathrm{K}$. The inset shows a small van der Waals loop present around the critical density (see text). 


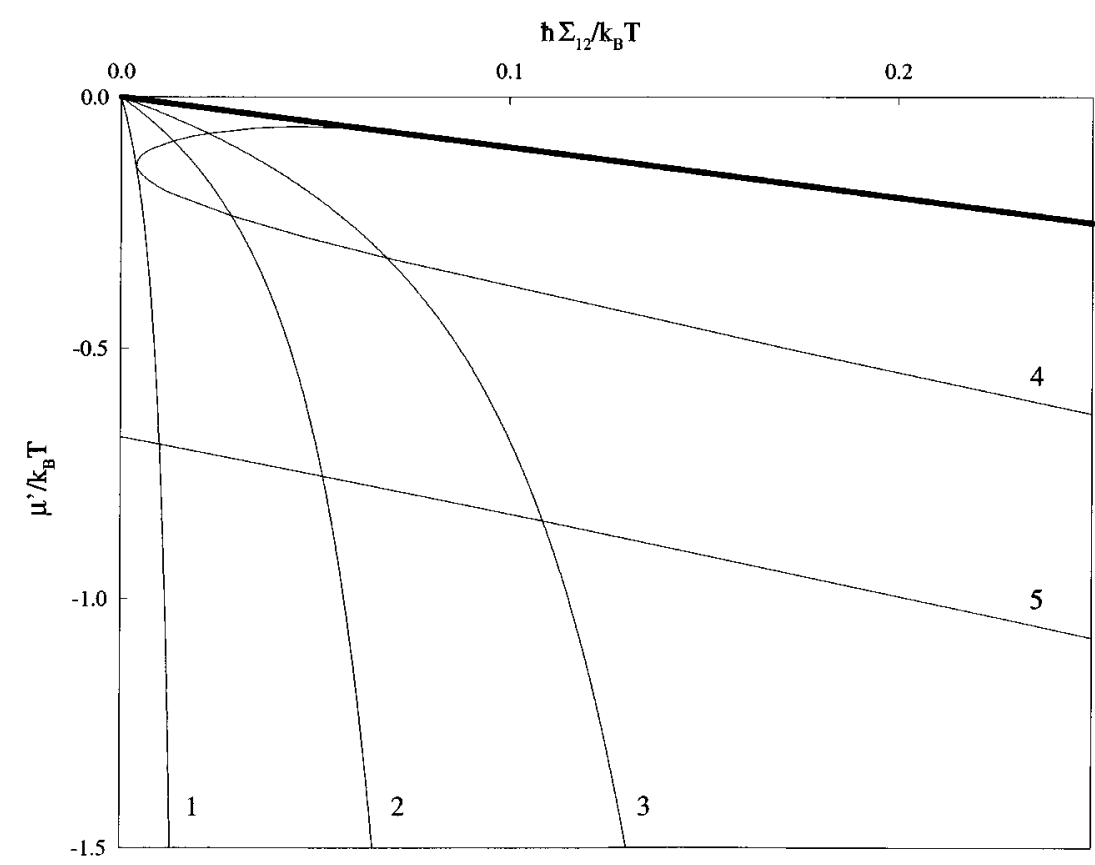

FIG. 8. Lines of constant chemical potential (4 and 5, the first having the largest chemical potential), and solution curves of the equation $\hbar \Sigma_{12}=n_{\mathbf{0}} T^{\mathrm{MB}}(\mathbf{0 , 0 , 0} ; 0)$ in the $\mu^{\prime}-\hbar \Sigma_{12}$ plane for (1) $n_{0} a^{2}=10^{-5}$, (2) $n_{0} a^{2}=5 \times 10^{-5}$, and (3) $n_{0} a^{2}=10^{-4}$. Also indicated is the HugenholtzPines line.

approach is of a mean-field type, since no fluctuations in the background field have been taken into account. Fluctuations, and in particular phase fluctuations associated with vortices, should reduce the real critical temperature and result in a universal jump of the superfluid density. This is schematically shown in Fig. 11, where we plot the behavior of the superfluid density versus temperature. Only for temperatures larger than $T_{c}^{\mathrm{MF}}$, which is itself larger than $T_{c}^{\mathrm{KT}}$, at which the Nelson-Kosterlitz criterion $n_{s} \Lambda^{2}=4$ is satisfied, can we apply our theory.

We would like to point out that $T_{c}^{\mathrm{MF}}$ cannot be found by a straightforward application of perturbation theory around the ideal Bose gas. Indeed, calculating the free energy $F$ and the (normal) self-energy in the ladder approximation would result in

$$
\mu^{\prime}=\mu-\hbar \Sigma_{11}=\mu-2 n T^{\mathrm{MB}}(\mathbf{0 , 0}, \mathbf{0} ; 0)
$$

and in the equation of state

$$
n=\sum_{\mathbf{k}} N\left(\epsilon_{\mathbf{k}}-\mu^{\prime}\right)
$$

where the latter is now obtained by a minimization of the free energy. At a fixed temperature, and density, we first of all determine $\mu^{\prime}$ from Eq. (34) and subsequently $\mu$ from Eq. (33). Clearly, this calculation can be performed at any temperature, and we just obtain the ideal gas result where a phase transition occurs only at zero temperature. Notice, however, that for temperatures larger than $T_{c}^{\mathrm{MF}}$ the above

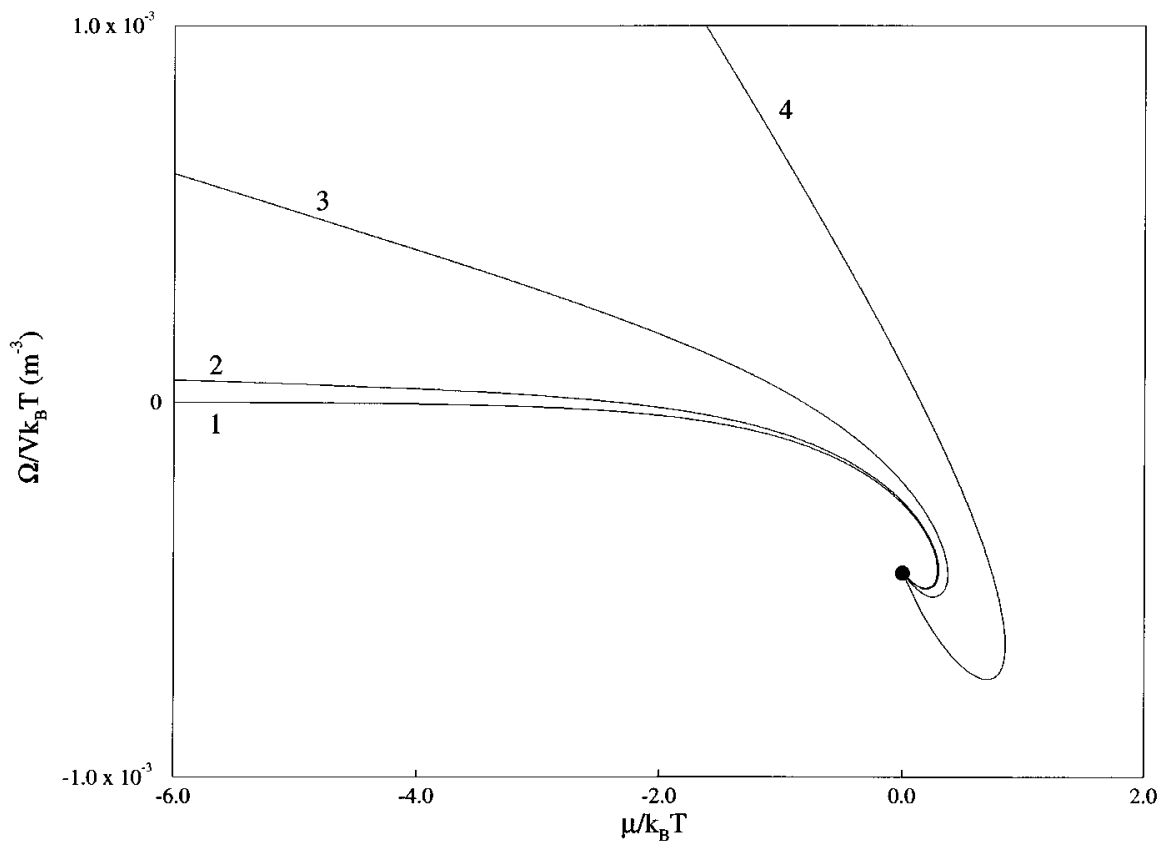

FIG. 9. Thermodynamic potential vs chemical potential at a temperature of $50 \mathrm{mK}$ and (1) $n_{0} a^{2}=0$, (2) $n_{0} a^{2}=10^{-5}, \quad$ (3) $n_{0} a^{2}=10^{-4}$, and (4) $n_{0} a^{2}=5$ $\times 10^{-4}$. The dot indicates the starting point of the corresponding curves in the $\mu^{\prime}-\hbar \Sigma_{12}$ plane, which lies in the origin for all values of $n_{0} a^{2}$. 


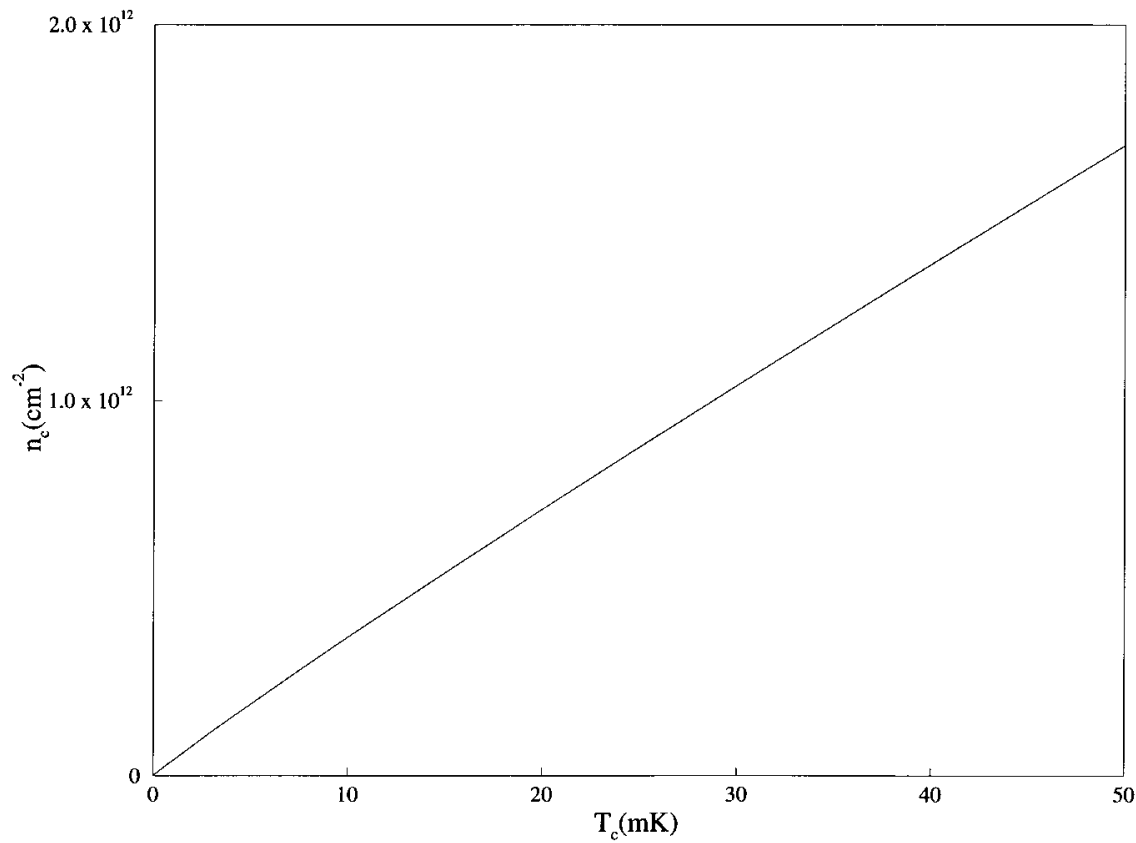

FIG. 10. Critical density vs temperature found from the criterion $\mu=0$, the point at which the variational approach signals the occurrence of an instability. procedure of minimizing the free energy $F$ leads, as expected, to exactly the same results as our minimization of the thermodynamic potential.

Finally, in Fig. 12 we show the thermodynamic potential as a function of condensate density for a fixed chemical potential in the case of zero temperature where a real condensate can exist in the two-dimensional system. In this case the solution curves of $\hbar \Sigma_{12}=n_{\mathbf{0}} T^{\mathrm{MB}}(\mathbf{0 , 0}, \mathbf{0} ; 0)$ do not start out in the origin of the $\mu^{\prime}-\hbar \Sigma_{12}$ plane, but essentially behave as depicted in Fig. 2, because without thermal fluctuations there are no infrared divergences when $\mu^{\prime}=-\hbar \Sigma_{12}$. Again in this extreme situation we find that the Hugenholtz-Pines relation is not satisfied, which, as in three dimensions, is due to the neglect of the coherence factors $u_{\mathbf{k}}$ and $v_{\mathbf{k}}$ in the equation for the many-body $T$ matrix by our variational approach.

\section{CONCLUSIONS}

We performed a variational calculation for a weakly interacting Bose gas, and applied this method to spin-polarized atomic hydrogen in both the three- and two-dimensional cases. We showed that the variational approach does not automatically lead to a consistent description of the system under study, and that the number of diagrams that is being summed can be rather restricted. In particular, the normal self-energy is obtained in the one-loop approximation only. The anomalous self-energy contains the ladder diagrams. We consequently improved all relevant expressions to the $T$ matrix level by using not the microscopic Hamiltonian, but an effective Hamiltonian to describe the Bose gas.

In three dimensions, this approach resolves the problem

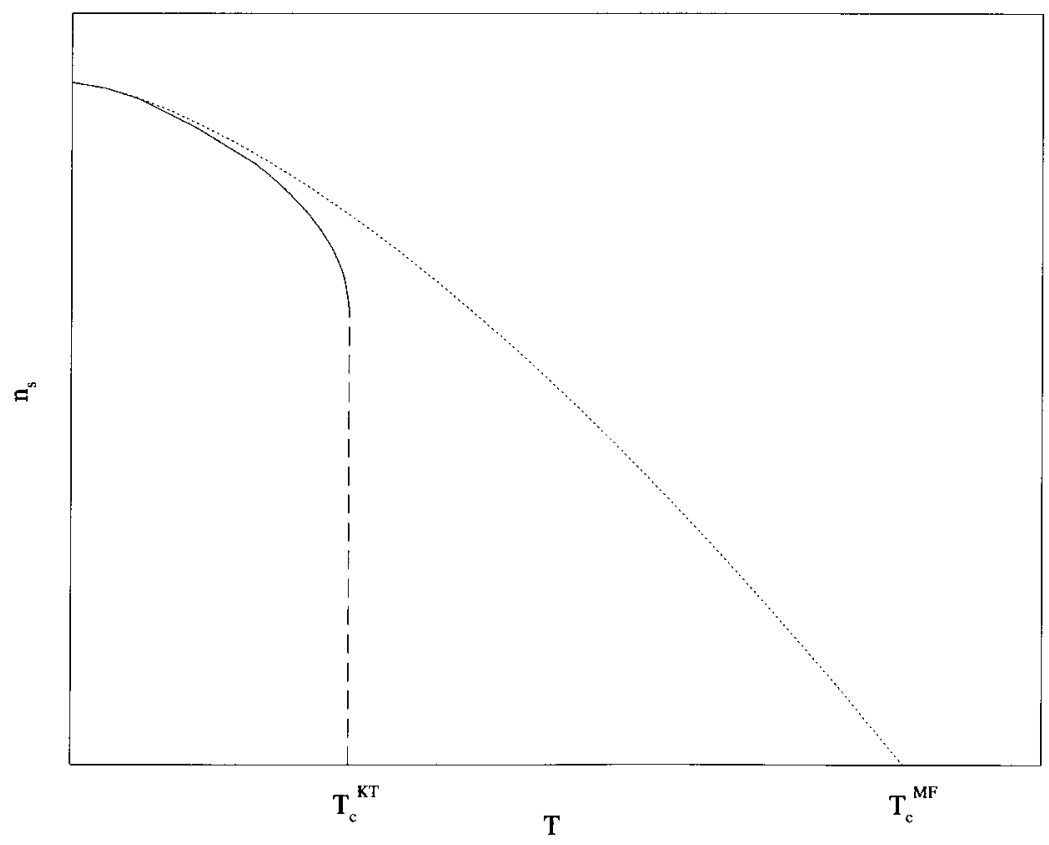

FIG. 11. Schematic behavior of superfluid density vs temperature. The solid line results from a renormalization-group analysis, that leads to the exact critical temperature $T_{c}^{\mathrm{KT}}$, obeying $n_{s} \Lambda_{c}^{2}=4$. The dashed curve corresponds to a mean-field result with a critical temperature $T_{c}^{\mathrm{MF}}$. Below $T_{c}^{\mathrm{MF}}$ the variational approach does not give information about the properties of the system. 


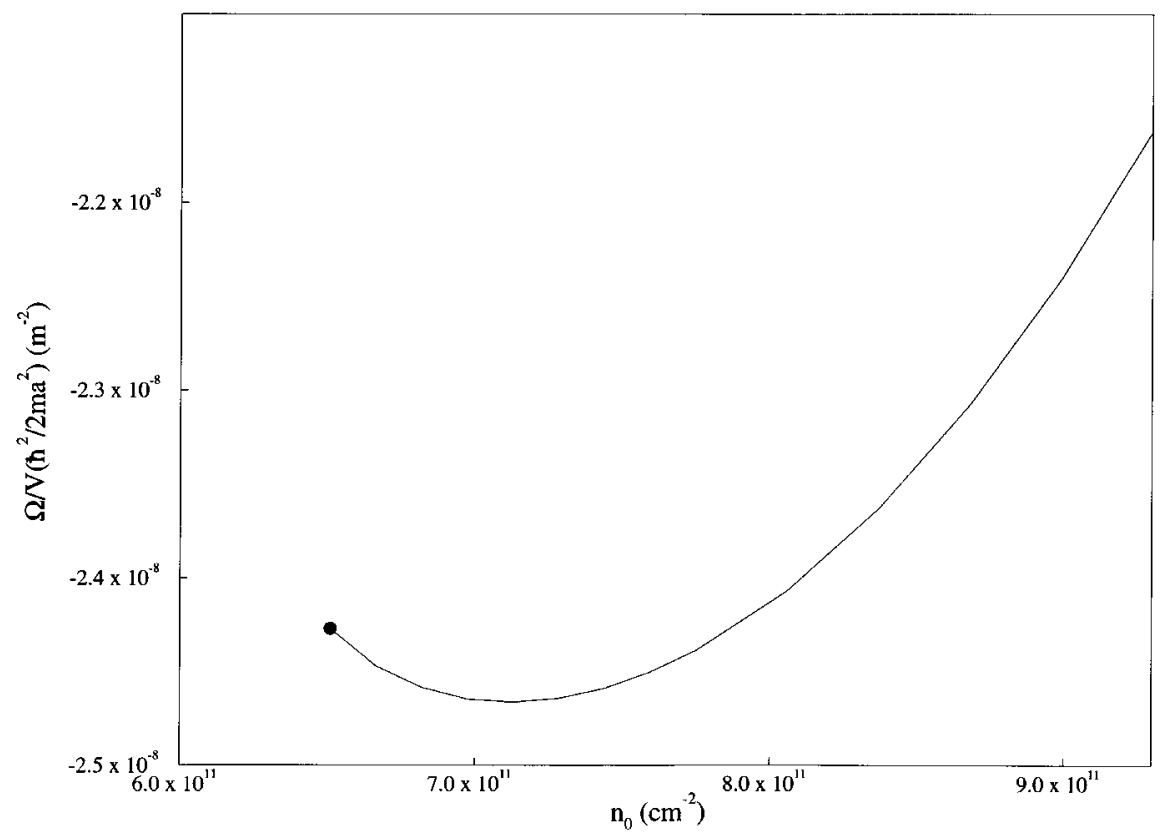

FIG. 12. Thermodynamic potential vs condensate density for fixed chemical potential at zero temperature, showing that the Hugenholtz-Pines condition, valid only at the end point of the curve, is not satisfied in the minimum of this curve. of double valuedness in the equation of state, that leads to the conclusion of a first-order phase transition, and is found in all previous approaches to the dilute Bose gas. The essential ingredient to resolve this problem is the use of the manybody $T$ matrix, which takes into account the fact that two particles collide in a medium, and not in a vacuum. To the best of our knowledge, this work comprises the first attempt for a dilute gas to include this effect of the medium. The (small) van der Waals loop we still find in the $p-n^{-1}$ diagram is a result of the neglect of the momentum and energy dependence of the $T$ matrix. The simplification in the coherence factors $u_{\mathbf{k}}$ and $v_{\mathbf{k}}$ leads to an overestimate of the effective interaction, and explains the fact that, in extreme conditions, far below the critical temperature, the HugenholtzPines relation is not satisfied. Using the full many-body $T$ matrix $T^{\mathrm{MB}}\left(\mathbf{k}, \mathbf{k}^{\prime}, \mathbf{K} ; z\right)$ will, however, resolve these issues. We also calculated condensate fraction as a function of temperature.

In two dimensions the variational approach, having a mean-field nature, signals only the onset of an instability in the theory, but unfortunately cannot describe the phase below the critical temperature. This is connected to the fact that within this variational approach it is not possible to find solutions where the dispersion relation of the quasiparticles displays a gap, playing the same role as the infrared cutoff in the theory with a quasicondensate, and enabling us to describe the system also below the critical temperature. The critical temperature we can infer from the occurence of the instability is an overestimate of the true critical temperature due to the neglect of the crucial phase fluctuations. In this respect it is important to note that introducing a quasicondensate $n_{0}\left(k_{0}\right)$, followed by a diagrammatic calculation of the equation of state [12], does give correct information about the Kosterlitz-Thouless phase, and thus incorporates the essential physics of the superfluid state. This in contrast to the mean-field variational approach presented here.

Finally we want to remark that solving the problem of the dilute Bose gas in the regime $n a \Lambda^{2} \gg 1$ using the full $T$ matrix Eq. (18) is a highly nontrivial task due to infrared difficulties even in three dimensions. A renormalizationgroup study should solve these difficulties and, more importantly, should also accurately describe the critical region. A study along these lines is underway.

\section{ACKNOWLEDGMENTS}

We would like to thank P.M. Stevenson for helpful discussions that introduced us to the variational approach to quantum field theory. We would also like to thank Ike Silvera for the hospitality enjoyed by one of us (M.B.) during his stay at Harvard University, and for interesting discussions concerning the ongoing experiments aimed at the observation of the Kosterlitz-Thouless transition in atomic hydrogen.
[1] N. N. Bogoliubov, J. Phys. (Moscow) 11, 23 (1947)

[2] For a review, we refer to the papers by T. J. Greytak and D. Kleppner, in New Trends in Atomic Physics, edited by C. Grynberg and R. Stora (North-Holland, Amsterdam, 1984), p. 1125; and I. F. Silvera and J. T. M. Walraven, in Progress in Low Temperature Physics, edited by D. F. Brewer (NorthHolland, Amsterdam, 1986), Vol. 10, p. 139. See also J. Opt.
Soc. Am. B 6 (11) (1989), special issue on laser cooling and atom trapping, edited by S. Chu and C. Wieman.

[3] A. Griffin, Physica C 156, 12 (1988).

[4] H. Shi, G. Verechaka and A. Griffin, Phys. Rev. B 50, 1119 (1994).

[5] T. D. Lee and C. N. Yang, Phys. Rev. 112, 1419 (1958).

[6] L. Reatto and J. P. Straley, Phys. Rev. 183, 321 (1969). 
[7] D. O. Edwards and I. B. Mantz, J. Phys. (Paris) Colloq. 41, C7-257 (1980).

[8] J. M. Kosterlitz and D. J. Thouless, J. Phys. C 6, 1181 (1973).

[9] D. R. Nelson and J. M. Kosterlitz, Phys. Rev. Lett. 39, 1201 (1977).

[10] V. N. Popov, Theor. Math. Phys. 11, 565 (1972); Functional Integrals in Quantum Field Theory and Statistical Physics (Reidel, Dordrecht, 1983), Chap. 6.

[11] D. S. Fisher and P. C. Hohenberg, Phys. Rev. B 37, 4936 (1988).

[12] H. T. C. Stoof and M. Bijlsma, Phys. Rev. E 47, 939 (1993).

[13] I. F. Silvera, in Bose-Einstein Condensation, edited by A. Griffin, D. W. Snoke, and S. Stringari (Cambridge University Press, Cambridge, 1995), p. 160.

[14] B. V. Svistunov, T. W. Hijmans, G. V. Shlyapnikov, and J. T. M. Walraven, Phys. Rev. B 43, 13412 (1991).

[15] A. I. Safonov, S. A. Vasilyev, I. S. Yasnikov, I. I. Lukashevich, and S. Jaakkola, Pis'ma Zh. Éksp. Teor. Fiz. 61, 998 (1995) [JETP Lett. 61, 1032 (1995)].

[16] A. Matsubara, T. Arai, S. Hotta, J. S. Korhonen, T. Mizusaki, and A. Hirai, in Bose-Einstein Condensation (Ref. [13]), p. 478.

[17] W. Petrich, M. H. Anderson, J. R. Ensher, and E. A. Cornell, Phys. Rev. Lett. 74, 3352 (1995).

[18] K. B. Davis, M. O. Mewes, M. A. Joffe, M. R. Andrews, and W. Ketterle, Phys. Rev. Lett. 74, 5202 (1995).

[19] M. H. Anderson, J. R. Ensher, M. R. Matthews, C. E. Wieman, and E. A. Cornell, Science 269, 198 (1995).

[20] C. C. Bradley, C. A. Sackett, J. J. Tollett, and R. G. Hulet, Phys. Rev. Lett. 75, 1687 (1995).

[21] K. B. Davis, M. O. Mewes, M. R. Andrews, N. J. van Druten, D. S. Durfee, D. M. Kurn, and W. Ketterle, Phys. Rev. Lett. 75, 3969 (1995).

[22] L. V. Butov, A. Zrenner, G. Abstreiter, G. Böhm, and G. Weimann, Phys. Rev. Lett. 73, 304 (1994)

[23] D. W. Snoke and J. P. Wolfe, Phys. Rev. B 42, 7876 (1990), and references therein.
[24] D. W. Snoke, J. P. Wolfe, and A. Mysyrowicz, Phys. Rev. Lett. 64, 2543 (1990); J. L. Lin and J. P. Wolfe, Phys. Rev. Lett. 71, 1222 (1993).

[25] L. I. Schiff, Phys. Rev. 130, 458 (1963).

[26] G. Rosen, Phys. Rev. 173, 1680 (1968).

[27] P. M. Stevenson, Phys. Rev. D 32, 1389 (1985).

[28] W. A. Bardeen and M. Moshe, Phys. Rev. D 28, 1372 (1983).

[29] Y. Zhang, M. Zhou, and B. Xu, Phys. Rev. B 47, 898 (1993).

[30] N. D. Mermin, Phys. Rev. 176, 250 (1968); N. D. Mermin and H. Wagner, Phys. Rev. Lett. 22, 1133 (1966); P. C. Hohenberg, Phys. Rev. 158, 383 (1967).

[31] See, for example, J. W. Negele and H. Orland, Quantum Many-Particle Systems (Addison-Wesley, New York, 1988).

[32] A. L. Fetter and J. D. Walecka, Quantum Theory of ManyParticle Systems (McGraw-Hill, New York, 1971), Chap. 10.

[33] N. M. Hugenholtz and D. Pines, Phys. Rev. 116, 489 (1958).

[34] H. T. C. Stoof, Phys. Rev. A 49, 3824 (1994).

[35] P. A. Ruprecht, M. J. Holland, K. Burnett, and M. Edwards, Phys. Rev. A 51, 4704 (1995).

[36] B. J. Verhaar, K. Gibble, and S. Chu, Phys. Rev. A 48, R3429 (1993).

[37] A. J. Moerdijk, W. C. Stwalley, R. G. Hulet, and B. J. Verhaar, Phys. Rev. Lett. 72, 40 (1994).

[38] H. T. C. Stoof, L. P. H. de Goey, W. M. H. M. Rovers, P. S. M. Kop Jansen, and B. J. Verhaar, Phys. Rev. A 38, 1248 (1988).

[39] T. Barnes and G. I. Ghandour, Phys. Rev. D 22, 924 (1980).

[40] K. Huang and C. N. Yang, Phys. Rev. 105, 767 (1957).

[41] H. T. C. Stoof, J. M. V. A. Koelman, and B. J. Verhaar, Phys. Rev. B 38, 4688 (1988).

[42] J. P. H. W. van den Eijnde, C. J. Reuver, and B. J. Verhaar, Phys. Rev. B 28, 6309 (1983).

[43] J. Zinn-Justin, Quantum Field Theory and Critical Phenomena (Oxford University Press, New York, 1989).

[44] H. T. C. Stoof, M. Bijlsma, and M. Houbiers, J. Res. Natl. Inst. Stand. Technol. (to be published). 